\title{
Growth Order of Eigenfunctions of Schrödinger Operators with Potentials Admitting Some Integral Conditions I - General Theory -
}

\author{
By
}

\author{
Masaharu ARAi* and Jun UChIYAmA**
}

\begin{abstract}
In this paper we consider the sharp estimates of the growth orders of the eigenfunctions of the Schrödinger operators with potentials oscillating violently at infinity. We make use of the modified Kato's method (Comm. Pure Appl. Math., 12 (1959), 403-425) and we apply the ideas of J. Uchiyama and O. Yamada (Publ. RIMS, Kyoto Univ., 26 (1990), 419-449). Applications will be given in the next paper [2] in this issue.
\end{abstract}

\section{§o. Introduction}

Let us consider the lower bounds of the growth order of a solution $u(x) \in$ $H_{l o c}^{2}(\Omega)$ of a second order elliptic equation

$$
-\sum_{j=1}^{n}\left(\partial_{j}+\sqrt{-1} b_{j}(x)\right)^{2} u(x)+\left(q_{1}(x)+q_{2}(x)\right) u(x)=0
$$

for $x \in \Omega:=\left\{x\left|x \in \boldsymbol{R}^{n},\right| x \mid>R_{0}\right\}$, where $\partial_{j}=\partial /\left(\partial x_{j}\right), b_{j}(x)$ and $q_{1}(x)$ are realvalued functions, $q_{2}(x)$ is a complex-valued function. To obtain the lower bounds of the growth order is one of main tools to show the non-existence of eigenvalues embedded in the continuous spectrum.

There are, roughly speaking, three methods to obtain the lower bounds of the growth order of a solution of $(0.1)$. The first is the method given by Kato [6], by which we aim to have the result similar to Theorem 1.1 in $\$ 1$ straightly. The second is the one given by Agmon [1], by which we aim to have the differential inequality yielding the result similar to Theorem 1.1. The last is the one given by Roze [10] and Eidus [4], by which we aim to show the result similar to Theorem 1.1 by contradiction. Making reference to Eastham-Kalf [3,

Communicated by T. Kawai, January 11, 1995.

1991 Mathematical Subject Classification : Primary 35B05; Secondary 35J15

${ }^{*}$ Department of Mathematics and Physics, Faculty of Science and Engineering, Ritsumei-

kan University, Kyoto 603, Japan.

${ }^{* *}$ Kyoto Institute of Technology, Matsugasaki, Sakyo-ku, Kyoto 606, Japan. 
pp. $170-185$, pp. 203-204], so far the Kato's method had the most obstacles and the Roze-Eidus' method was most powerful but also most sophisticated. First Uchiyama [11] (Kato's method) and Mochizuki [8] (Roze-Eidus' method) manifested the differences between the results which were gained by the applications of the different methods to the same problem. In this paper we employ the Kato's method and modify it in many elaborate points of proving Theorem 1.1. In Arai-Uchiyama [2] we can cover the results given by Uchiyama-Yamada [13] which based on the Roze-Eidus' method. As a conclusion, we have completely removed the hurdles which were lying in the application of the Kato's method.

As an application of our results we can generalize the results of Khosrovshahi-Levine-Payne [7] and Kalf-Kumar [5], which treated the Schrödinger operators with potentials admitting some integral conditions. We can also generalize the result of Agmon [1]. In the next article Arai-Uchiyama [2], we will give these results. We will also apply our theory to von NeumannWigner [9] example in other article.

We would like to express our gratitude to Professor A. Iwatsuka of Kyoto University, who kindly discussed Lemma 5.2 with us.

\section{§1. Assumptions and Main Results}

We list up the notations used in this paper, which are about the same as given in Uchiyama-Yamada [13].

\section{Notations.}

$$
\langle\xi, \eta\rangle=\xi_{1} \eta_{1}+\cdots+\xi_{n} \eta_{n} \text { for } \xi={ }^{t}\left(\xi_{1}, \cdots, \xi_{n}\right), \eta={ }^{t}\left(\eta_{1}, \cdots, \eta_{n}\right) \in \mathbb{C}^{n},
$$

where superscript $t$ means the transposed form of the corresponding quantity ;

$|\xi|=(\langle\xi, \bar{\xi}\rangle)^{1 / 2}$ for $\xi \in C^{n}$;

$\widehat{x}=x /|x|$ and $r=|x|$ for $x={ }^{t}\left(x_{1}, \cdots, x_{n}\right) \in \mathbb{R}^{n}$;

$\partial_{j}=\partial / \partial x_{j}$ and $\partial_{r}=\partial / \partial r$;

$D_{j}=\partial_{j}+\sqrt{-1} b_{j}(x), D={ }^{t}\left(D_{1}, \cdots, D_{n}\right)$ and $D_{r}=\langle\hat{x}, D\rangle$;

$f^{\prime}(r)=(d / d r) f(r)$ and $f^{\prime \prime}(r)=\left(d^{2} / d r^{2}\right) f(r)$;

$\nabla f={ }^{t}\left(\partial_{1} f, \cdots, \partial_{n} f\right)$ for a scalar-valued function $f(x)$;

$B=B(x)=\operatorname{curl} b(x)=\left(B_{j k}(x)\right)$ is an $n \times n$ matrix whose $(j, k)$-element is $B_{j k}(x):=\partial_{j} b_{k}(x)-\partial_{k} b_{j}(x)$;

$(f)_{ \pm}(x)=\max \{0, \pm f(x)\} \geq 0$ for a real-valued function $f(x)$;

$\operatorname{supp}[f]$ denotes the closure of $\{x \mid f(x) \neq 0\}$;

$C^{j}(\Omega)$ denotes the class of $j$-times continously differentiable functions ; 
$C_{0}^{j}(\Omega)=\left\{f(x) \in C^{j}(\Omega) \mid \operatorname{supp}[f]\right.$ is a compact set in $\left.\Omega\right\}$;

$C_{0}^{\infty}(\Omega)=\bigcap_{j=1}^{\infty} C_{0}^{j}(\Omega)$;

$H^{m}(\Omega)$ denotes the class of $L^{2}$-functions in $\Omega$ such that all distributional derivatives up to order $m$ belong to $L^{2}(\Omega)$;

$H_{l o c}^{m}(\Omega)$ denotes the class of $L_{l o c}^{2}$-functions in $\Omega$ such that all distributional derivatives up to order $m$ belong to $L_{l o c}^{2}(\Omega)$;

$$
\left(\int_{|x|=t}-\int_{|x|=s}\right) f(x) d S=\int_{|x|=t} f(x) d S-\int_{|x|=s} f(x) d S .
$$

Next we shall state the conditions required in the Theorems.

Assumptions. Let us consider the equation (0.1). We assume that there exist some real-valued functions $\psi_{i}(r) \in C^{0}\left[R_{0}, \infty\right), \sigma_{i}(r), \eta_{i}(r) \in C^{1}\left[R_{0}, \infty\right)(i$ $=1,2), \tau(r) \in C^{0}\left[R_{0}, \infty\right)$ and some constants $a_{i}(i=1,2)$ satisfying the following conditions (B.1)-(F.2) :

(B.1) each $b_{j}(x)$ is a real-valued function;

(B.2) for any $w(x) \in H_{\text {loc }}^{1}(\Omega)$ we have $b_{j}(x) w(x),\left(\partial_{j} b_{k}(x)\right) w(x) \in L_{l o c}^{2}(\Omega)$;

(C.1) $q_{1}(x)$ is a real-valued function;

(C.2) for any $w(x) \in H_{\text {loc }}^{1}(\Omega)$ we have $q_{1}(x)|w(x)|^{2} \in L_{l o c}^{1}(\Omega)$;

(C.3) for any $w(x) \in H_{\text {loc }}^{1}(\Omega)$ we have $\partial_{r} q_{1}(x) \cdot|w(x)|^{2} \in L_{l o c}^{1}(\Omega)$;

(D.1) $q_{2}(x)$ may be a complex-valued function ;

(D.2) for any $w(x) \in H_{l o c}^{1}(\Omega)$ we have $q_{2}(x)|w(x)|^{2} \in L_{l o c}^{1}(\Omega)$;

(E.1) $\sigma_{i}(r)$ is bounded in $\left[R_{0}, \infty\right)$ and $\sigma_{i}(r)>0(i=1,2)$ for any $r \geq R_{0}$;

(E.2) $\quad \eta_{i}(r)$ is bounded in $\left[R_{0}, \infty\right)$ and we have for any $r \geq R_{0}$

$$
\eta_{i}(r)<2(i=1,2) \text {; }
$$

(E.3) $\quad \phi_{i}(r)>0(i=1,2)$ for any $r \geq R_{0}$;

(E.4) $\lim _{r \rightarrow \infty} r^{-1} \phi_{i}(r) \sigma_{i}(r)^{-1}=0(i=1,2)$;

(E.5) $r\left\{\sigma_{1}^{\prime}(r)-\sigma_{2}^{\prime}(r)-\left(\eta_{1}^{\prime}(r)-\eta_{2}^{\prime}(r)\right)\right\}$ is bounded in $\left[R_{0}, \infty\right)$;

(E.6) there exists some constant $C_{1} \geq 1$ such that for any $r \geq R_{0}$

$$
C_{1}^{-1} \sigma_{2}(r) \leq \tau(r) \leq C_{1}
$$

(E.7) $\lim _{r \rightarrow \infty} \psi_{2}(r)^{2} \sigma_{2}(r)^{-1} \exp \left(\int_{R_{0}}^{r} \frac{\tau(t)-\eta_{2}(t)}{t} d t\right)=0$;

(F.1) $\quad a_{1}>1, a_{2}>0$;

(F.2) $\quad \limsup _{r \rightarrow \infty} \psi_{i}(r)^{2} \sigma_{i}(r)^{-1}\left[r \partial_{r} q_{1}+\eta_{i}(r) q_{1}+a_{i} \sigma_{i}(r)^{-1}\left|r q_{2}-Q_{i}^{\prime}(r)\right|^{2}\right.$

$$
\left.+\left(2-\eta_{i}(r)\right)^{-1}|B x|^{2}\right]<0(i=1,2),
$$

where we put 


$$
Q_{i}(r)=4^{-1}\left(\eta_{i}(r)-\sigma_{i}(r)\right)
$$

Our main results are as follows :

Theorem 1.1. Let $u(x) \in H_{\text {loc }}^{2}(\Omega)$ satisfy the equation (0.1). Assume that (B)-(F) hold. If $\operatorname{supp}[u]$ is not a compact set in $\bar{\Omega}$, then we have

$$
\liminf _{R \rightarrow \infty} \Phi_{1}(R) \int_{|x|=R}\left[\left|D_{r} u\right|^{2}+\left\{r^{-2}+\left(q_{1}\right)_{-}\right\}|u|^{2}\right] d S>0,
$$

where

$$
\Phi_{1}(r)=\exp \left(\int_{R_{0}}^{r} \frac{\sigma_{1}(t)+\eta_{1}(t)}{2 t} d t\right)
$$

Theorem 1.2. In addition to the assumptions of Theorem 1.1, assume that

(G) there exist some real-valued bounded function $Q_{0}(x) \in C^{1}(\bar{\Omega})$, some positive and non-decreasing function $p(r) \in C^{1}\left[R_{0}, \infty\right)$ and some constant $0<$ $d_{1}<1$ satisfying the following (G.1)-(G.3) :

(G.1) for any $w(x) \in C_{0}^{\infty}(\Omega)$ we have

$$
\begin{aligned}
& \int_{\Omega}\left\{\left(q_{1}\right)_{-}(x)+\left(\operatorname{Re}\left[q_{2}\right]-r^{-1} \partial_{r} Q_{0}\right)_{-}(x)\right\}|w(x)|^{2} d x \\
& \quad \leq \int_{\Omega}\left\{d_{1}|\nabla w(x)|^{2}+p(r)|w(x)|^{2}\right\} d x
\end{aligned}
$$

(G.2) $\liminf _{r \rightarrow \infty} p(r) p(r+1)^{-1}>0$;

(G.3) $p^{\prime}(r)^{2} p(r)^{-3}$ is bounded in $\left[R_{0}, \infty\right)$.

Then we have

(1) $\quad \liminf _{R \rightarrow \infty} p(R) \Phi_{1}(R) \int_{R<|x|<R+1}|u(x)|^{2} d x>0$;

(2) moreover if $\int_{R_{0}}^{\infty} p(R)^{-1} \Phi_{1}(R)^{-1} d R=\infty$ then $u(x) \notin L^{2}(\Omega)$.

A simple example of a positive and non-decreasing function $p(r) \in$ $C^{1}\left[R_{0}, \infty\right)$ satisfying (G.2) and (G.3) is

$$
p(r)=\text { Const } r^{\delta}(\delta \geq 0)
$$

So we have

Corollary 1.3. In addition to the assumptions of Theorem 1.1, assume that

(G) there exist some real-valued bounded function $Q_{0}(x) \in C^{1}(\bar{\Omega})$, some 
constants $0<d_{1}<1$ and $\delta \geq 0$ such that for any $w(x) \in C_{0}^{\infty}(\Omega)$ we have

$$
\begin{aligned}
\int_{\Omega}\left\{\left(q_{1}\right)_{-}(x)+\right. & \left.\left(\operatorname{Re}\left[q_{2}\right]-r^{-1} \partial_{r} Q_{0}\right)_{-}(x)\right\}|w(x)|^{2} d x \\
& \leq \int_{\Omega}\left\{d_{1}|\nabla w(x)|^{2}+\text { Const } r^{\delta}|w(x)|^{2}\right\} d x
\end{aligned}
$$

Then we have

(1) $\liminf _{R \rightarrow \infty} R^{\delta} \Phi_{1}(R) \int_{R<|x|<R+1}|u(x)|^{2} d x>0$;

(2) moreover if $\int_{R_{0}}^{\infty} R^{-\delta} \Phi_{1}(R)^{-1} d R=\infty$ then $u(x) \notin L^{2}(\Omega)$.

Remark 1.4. If $B(x) \equiv O(n \times n$ zero matrix $)$, then in Theorem 1.1 and Theorem 1.2 the Assumptions (E.2) can be replaced with the following (E.2)'. (E.2) $\eta_{i}(r)$ is bounded in $\left[R_{0}, \infty\right)$ and $\eta_{i}(r) \leq 2(i=1,2)$.

The rationalization of Remark 1.4 will be given in the proof of Lemma 4.1.

Remark 1.5. We remark that the assumption (E.7) is set for $i=2$ only, which requests $\eta_{2}(r)$ to be large. On the other hand $\Phi_{1}(r)$ in Theorem 1.1 concerns for $i=1$ only so that the smaller $\sigma_{1}(r)+\eta_{1}(r)$ we choose, the better estimate we have as the lower bound of the growth order of $u$ at infinity. So we separate the roles of $\eta_{1}(r)$ and $\eta_{2}(r)$. This idea was introduced in Uchiyama-Yamada [13]. For example, consider the case

$$
\left\{\begin{array}{l}
q_{1}(x)<0 \text { for } r \geq R_{0} \\
|B(x) x|^{2}=0(1) \cdot \psi_{1}(r)^{-2} \sigma_{1}(r) \text { as } r \rightarrow \infty \\
\sup _{r \geq R_{0}} \eta_{1}(r)<2
\end{array}\right.
$$

If (F.2) holds for $i=1$, then (F.2) holds for $i=2$ with $\psi_{2}(r)=\psi_{1}(r), \sigma_{2}(r)=$ $\sigma_{1}(r), \eta_{2}(r)=\eta_{1}(r)+\eta_{0}, a_{2}=a_{1}$, where $\eta_{0} \geq 0$ is a constant satisfying $\eta_{0}+\sup _{r \geq R_{0}}$ $\eta_{1}(r)<2$. (Under these choices there exists no contradiction in (E.1)-(E.5).) Thus even if (E.7) with $\eta_{2}(r)=\eta_{1}(r)$ does not hold, we can expect that (E.7) is satisfied by new $\eta_{2}(r)$ different from $\eta_{1}(r)$. The benefit from not necessarily assuming $\eta_{1}(r)=\eta_{2}(r)$ will be seen in Example 2.1, though it does not satisfy (1.2).

Remark 1.6. Let us consider the case

$$
\begin{aligned}
& r q_{2}(x)-Q_{i}^{\prime}(r)=\mathrm{O}(1) \cdot \psi_{i}(r)^{-1} \sigma_{i}(r) \text { as } r \rightarrow \infty(i=1,2), \\
& \left|B_{x}\right|^{2}=\mathrm{O}(1) \cdot \psi_{i}(r)^{-2} \sigma_{i}(r)\left\{2-\eta_{i}(r)\right\} \text { as } r \rightarrow \infty(i=1,2) .
\end{aligned}
$$

Then (F.1) can be replaced with

(F.1) $\quad a_{1}=1, a_{2}=0$. 
In fact the left side of (F.2) depends continuously on $a_{i}$ under our additional conditions. So (F.2) with (F.1)' leads us to (F.2) with (F.1).

The ideas applied to the proofs. Now, let us state our strategy. We introduce an auxilliary function $\rho(r) \in C^{2}\left[R_{0}, \infty\right)$ and put $v(x)=e^{\rho(|x|)} u(x)$. Then $v(x)$ satisfies a differential equation (3.1). Multiplying both side of (3.1) by a suitable quantity and integrating by parts in $\left(R_{0}<\right) s<\left|x_{1}\right|<t$, we have the identity (3.3). Preparing another identity (3.10) and adding them, we have the key lemma, i.e. Lemma 3.8, involving another auxilliary function $\phi(r)$.

Lemma 3.8 and Lemma 4.1 (1) show the non-decrease of $F_{1}(t ; 0,0 ; u)$ which is defined in Definition 3.7 and is related to the assumptions with the index $i=$ 1. Proposition 5.1 shows from the non-decrease of $F_{1}(t ; 0,0 ; u)$ that if there existed $R_{*} \geq R_{0}$ satisfying

$$
F_{1}\left(R_{*} ; 0,0 ; u\right)>0
$$

then Theorem 1.1 would hold. Thus if such $R_{*}$ existed then the auxilliary functions $\rho$ and $\phi$ and the Assumptions corresponding to the index $i=2$ were not necessary. But we cannot show this in general so we must continue our discussion.

Read $m_{0} \rho_{1}$ and $\rho_{2}$ in the text as $\rho$ and read $\phi_{0}$ in the text as $\phi$ here. It is obvious that one of the two cases stated in Lemma 6.1 occurs. In Case 1 of Lemma 6.1, by the proof of Lemma 6.2, Theorem 1.1 holds if we choose suitable $\rho^{\prime}$. In Case 2 of Lemma 6.1 , by the proof of Lemma 6.4, (1.3) and hence Theorem 1.1 would hold if

$$
F_{2}(r ; \rho, \phi ; v)>0 \text { for large } r
$$

and

(1.5) $\phi$ were so large compared to $r^{-1} \rho^{\prime}$ that (6.4) held.

On the other hand, by Lemma 4.10 , if we chose

(1.6) some large $\rho$,

and

(1.7) so small $\phi$ that Lemma 5.4 held,

then (1.4) would hold. But unfortunately there is no $\phi$ and $\rho$ satisfying the requirements (1.5)-(1.7). So we introduce two $\rho$ 's, denoted as $m_{0} \rho_{1}$ and $\rho_{2}$ in the text, where $\rho=m_{0} \rho_{1}$ satisfies (1.6) and $\rho_{2}$ is a smaller one. In Definition 5.3 we construct $\phi$, denoted as $\phi_{0}$ in the text, satisfying (1.5) with $\rho=\rho_{2}$ and (1.7). Then (1.6) and (1.7) and so (1.4) hold with $\rho=m_{0} \rho_{1}$. By Lemma 5.15, (1.4) with $\rho=m_{0} \rho_{1}$ implies (1.4) with $\rho=\rho_{2}$. The validity of (1.4) and (1.5) with $\rho=\rho_{2}$ implies (1.3) and our proof completes. 


\section{§2. Examples}

The usual eigenvalue problem

$$
-\sum_{j=1}^{n}\left(\partial_{j}+\sqrt{-1} b_{j}(x)\right)^{2} u(x)+\left(V_{1}(x)+V_{2}(x)+V_{3}(r)\right) u(x)=\lambda u(x)
$$

can be reduced to $(0.1)$ by putting $q_{1}(x)=V_{1}(x)-\lambda$ and $q_{2}(x)=V_{2}(x)+V_{3}(r)$, where $r=|x|$.

Let us state the meaning of $Q_{i}(r)$ in the Assumption (F.2) and $Q_{0}$ in the Assumption (G.1). If $V_{3}(r)$ in (2.1), namely a part of $q_{2}(x)$, oscillates violently at infinity but $Q(r)=\int_{R_{0}}^{r} t V_{3}(t) d t$ is gentle at infinity in the sense that $Q(r)$ is bounded, then by $Q^{\prime}(r)=r V_{3}(r)$ we can remove the influence of $V_{3}(r)$ to $q_{2}(x)$ as is seen in the Assumption (F.2) and (G.1). An example of $V_{3}(r)$ will be given in Remark 2.2.

The next example shows the benefit from not necessarily assuming $\eta_{1}(r)=$ $\eta_{2}(r)$ and the meaning of (F.2).

Example 2.1. Let us consider a solution $u(x)$ of the equation

$$
\begin{aligned}
-\sum_{j=1}^{n}\left(\partial_{j}\right. & \left.+\sqrt{-1} b_{j}(x)\right)^{2} u(x) \\
& +\left\{-h(x)+V_{1}(x)+V_{2}(x)+r^{-1} Q^{\prime}(r)\right\} u(x)=\lambda u(x)
\end{aligned}
$$

for $x \in \Omega:=\left\{x|| x \mid>R_{0}\right\}$ such that $\operatorname{supp}[u]$ is not a compact set in $\bar{\Omega}$.

We assume that there exists some constant $\beta \geq 0$ such that

$h(x) \in C^{1}(\bar{\Omega})$ is a positive homogeneous function of degree $\alpha>-2$; $V_{1}(x) \in C^{1}(\bar{\Omega})$ is a real-valued function satisfying

$$
V_{1}(x)=0\left(r^{\alpha}(\log r)^{-\beta}\right), \partial_{r} V_{1}(x)=\mathrm{o}\left(r^{\alpha-1}(\log r)^{-\beta}\right) \text { as } r \rightarrow \infty \text {; }
$$

$V_{2}(x) \in C^{0}(\bar{\Omega})$ is a complex-valued function satisfying

$$
V_{2}(x)=\mathrm{o}\left(r^{(\alpha / 2)-1}(\log r)^{-\beta}\right) \text { as } r \rightarrow \infty ;
$$

$Q(r) \in C^{1}(\bar{\Omega})$ is a real-valued function satisfying

$$
Q(r)=0\left((\log r)^{-\beta}\right) \text { as } r \rightarrow \infty ;
$$

$b_{j}(x) \in C^{1}(\bar{\Omega})$ is a real-valued function satisfying

$$
|B(x) x|=0\left(r^{(\alpha / 2)}(\log r)^{-(\beta / 2)}\right) \text { as } r \rightarrow \infty ;
$$

$\lambda$ is a constant satisfying $-\infty<\lambda<\infty$ for $\alpha>0$ or $\lambda=0$ for $-2<\alpha \leq 0$. (This condition means that $\lambda$ is small as compared with $h(x)$ near infinity.)

Then we have 


$$
\left\{\begin{array}{l}
\lim _{R \rightarrow \infty} R^{|\alpha| / 2} \exp \left\{\varepsilon(\log R)^{1-\beta}\right\} \int_{R<|x|<R+1}|u|^{2} d x=\infty \\
\text { for any } \varepsilon>0 \text { if } 0 \leq \beta<1 ; \\
\lim _{R \rightarrow \infty} R^{|\alpha| / 2}(\log R)^{\varepsilon} \int_{R<|x|<R+1}|u|^{2} d x=\infty \\
\begin{array}{ll}
\text { for any } \varepsilon>0 & \text { if } \beta=1 ; \\
\liminf _{R \rightarrow \infty} R^{|\alpha| / 2} \int_{R<|x|<R+1}|u|^{2} d x>0 & \text { if } \beta>1 ; \\
u(x) \notin L^{2}\left(|x|>R_{0}\right) & \text { if }|\alpha|<2, \beta \geq 0 \text { or } \alpha=2, \beta \geq 1 .
\end{array}
\end{array}\right.
$$

The case $-2<\alpha<0$ and $\lambda>0$ will be treated in Example 2.4 under weaker conditions.

Proof of Example 2.1. Let $q_{1}(x)=-h(x)+V_{1}(x)-\lambda$ and $q_{2}(x)=V_{2}(x)$ $+r^{-1} Q^{\prime}(r)$. Then (2.2) reduces to $(0.1)$. Now let us construct functions $\phi_{i}(r)$, $\sigma_{i}(r), \eta_{i}(r), \tau(r), Q_{0}(r)$ and constants $a_{i}(i=1,2)$ and $\delta$ satisfying the assumptions of Corollary 1.3. Let $\varepsilon \in\left(0,5^{-1}(2+\alpha)\right)$ be arbitrary. And let

$$
\begin{aligned}
& \phi_{i}(r)=r^{-(\alpha / 2)}(i=1,2), \\
& \sigma_{i}(r)=2 \varepsilon(\log r)^{-\beta}-2 Q(r)(i=1,2), \\
& \eta_{i}(r)=\varepsilon \delta_{i 2}-\alpha+2 \varepsilon(\log r)^{-\beta}+2 Q(r)(i=1,2), \\
& \tau(r) \equiv \tau_{0} \text { be a constant satisfying } 0<\tau_{0}<\varepsilon, \\
& Q_{0}(r)=Q(r), \\
& a_{1}>1, a_{2}>0 \text { be arbitrary constants, } \\
& \delta=\max \{0, \alpha\} .
\end{aligned}
$$

By $Q(r)=\mathrm{o}\left((\log r)^{-\beta}\right)$, there exists some $R_{1} \geq R_{0}$ such that for any $r \geq R_{1}$ we have $|Q(r)|<2^{-1} \varepsilon(\log r)^{-\beta}$ and $\log r \geq 1$. Then for any $r \geq R_{1}$

$$
\begin{aligned}
& \varepsilon(\log r)^{-\beta}<\sigma_{i}(r)<3 \varepsilon(\log r)^{-\beta} \leq 3 \varepsilon<2+\alpha-2 \varepsilon \\
& \varepsilon \delta_{i 2}-\alpha+\varepsilon(\log r)^{-\beta}<\eta_{i}(r)<\varepsilon-\alpha+3 \varepsilon(\log r)^{-\beta} \leq 4 \varepsilon-\alpha<2-\varepsilon .
\end{aligned}
$$

So we can easily verify that the conditions (E.1)-(E.6) hold.

By $\eta_{2}(t) \geq \varepsilon-\alpha$ for $t \geq R_{1}$, we have for $r \geq R_{1}$

$$
\psi_{2}^{2} \sigma_{2}^{-1} \exp \left(\int_{R_{0}}^{r} \frac{\tau(t)-\eta_{2}(t)}{t} d t\right) \leq \mathrm{Const}(\log r)^{\beta} r^{\tau_{0}-\varepsilon}
$$

which leads us to (E.7).

Now we check (F.2). Note that $Q_{i}(r)=4^{-1}\left(\eta_{i}-\sigma_{i}\right)=Q(r)+4^{-1}\left\{\varepsilon \delta_{i 2}-\alpha\right\}$. We have

$$
\begin{gathered}
\psi_{i}^{2} \sigma_{i}^{-1}\left\{r \partial_{r} q_{1}+\eta_{i} q_{1}+a_{i} \sigma_{i}^{-1}\left|r q_{2}-Q_{i}^{\prime}\right|^{2}+\left(2-\eta_{i}\right)^{-1}|B x|^{2}\right\} \\
=r^{-\alpha} \sigma_{i}^{-1}\left[-\left(\alpha+\eta_{i}\right) h(x)-\eta_{i} \lambda+\left(r \partial_{r} V_{1}+\eta_{i} V_{1}\right)\right. \\
\left.+a_{i} \sigma_{i}^{-1}\left|r V_{2}\right|^{2}+\left(2-\eta_{i}\right)^{-1}|B x|^{2}\right] .
\end{gathered}
$$

Therefore, by $\left(\alpha+\eta_{i}\right) \sigma_{i}^{-1} \geq \varepsilon(\log r)^{-\beta} \cdot(3 \varepsilon)^{-1}(\log r)^{\beta}=3^{-1}$ and by $r^{-\alpha} h(x) \geq$ 
$\min \{h(x)|| x \mid=1\}>0$, we have (F.2).

Since we have $(\alpha / 2)-1<\alpha$ for $\alpha>-2$, we have for any $r \geq R_{1},\left(q_{1}\right)_{-}(x)$ $+\left(\operatorname{Re}\left[q_{2}\right]-r^{-1} Q^{\prime}\right)_{-}(x) \leq$ Const $r^{\alpha}$. Thus $(1.1)$ is satisfied by $\delta=\max \{0, \alpha\}$. Now we calculate

$$
\begin{aligned}
\Phi_{1}(R) & =\exp \left(\int_{R_{0}}^{R} \frac{\sigma_{1}(t)+\eta_{1}(t)}{2 t} d t\right) \\
& =\left(\frac{R}{R_{0}}\right)^{-(\alpha / 2)} \exp \left(2 \varepsilon \int_{R_{0}}^{R} t^{-1}(\log t)^{-\beta} d t\right) \\
& = \begin{cases}\text { Const } R^{-(\alpha / 2)} \exp \left\{\frac{2 \varepsilon}{1-\beta}(\log R)^{1-\beta}\right\} & \text { if } \beta \neq 1, \\
\text { Const } R^{-(\alpha / 2)}(\log R)^{2 \varepsilon} & \text { if } \beta=1 .\end{cases}
\end{aligned}
$$

Using the arbitrariness of $\varepsilon>0$, we can replace $2 \varepsilon(1-\beta)^{-1}$ (in case $0 \leq \beta<1$ ) or $2 \varepsilon$ (in case $\beta=1$ ) with $\varepsilon$. Thus by Corollary 1.3 and by $\delta-(\alpha / 2)=(|\alpha| / 2)$, we have the first three assertions, from which we can easily see the last assertion.

Remark 2.2. We give an example of $Q(r)$ satisfying the conditions given in Example 2.1. Let

$$
Q(r)=-\int_{r}^{\infty}(\log r)^{-\widetilde{\beta}} e^{r} \sin e^{r} d r
$$

for $\widetilde{\beta}>\beta$, where $\beta \geq 0$ is the one given in Example 2.1. Then we can see that

$$
r^{-1} Q^{\prime}(r)=r^{-1}(\log r)^{-\widetilde{\beta}} e^{r} \sin e^{r}
$$

oscillates violently at infinity but

$$
Q(r)=-(\log r)^{-\widetilde{\beta}} \cos e^{r}+\mathrm{o}\left(e^{-r}\right)=\mathrm{o}\left((\log r)^{-\beta}\right)
$$

is gentle at infinity

Remark 2.3. By Uchiyama-Yamada [13, Remark 1.8], there exists some non-trivial $u(x)$ satisfying

$$
\left\{\begin{array}{l}
-\Delta u-r^{\alpha} u=0(\alpha>-2) \text { in } \boldsymbol{R}^{n} \\
\limsup _{R \rightarrow \infty} R^{\alpha / 2} \int_{R<|x|<R+1}|u|^{2} d x<+\infty
\end{array}\right.
$$

Hence the above third estimate (, namely the one for $\beta>1$,) of lower bound of growth order of $u(x)$ is best possible for $\alpha \geq 0$.

Example 2.1 suggests us the followings.

(1) Since the terms linked with $q_{1}(x)$ only give the negativity of (F.2), $h(x)$ (, which is a main part of $q_{1}(x)$,) plays the leading role, and $V_{1}(x)$ (, which is a subordinate part of $\left.q_{1}(x)\right), q_{2}(x)$ and $B(x)$ play subordinate roles in (F.2). 
With the above Example, this facts reflects on $\Phi_{1}(r)$ through the choices of $\sigma_{1}(r)$ and $\eta_{1}(r)$, namely in $\Phi_{1}(r)$ the influence of $h(x)$, whose degree of homogenity is $\alpha$, is primary and the ones of $V_{1}(x), q_{2}(x)$ and $B(x)$ are the second.

(2) Moreover under the circumstance that we fix $h(x)$, we can see that the more gently $V_{1}(x), q_{2}(x)$ and $B(x)$ behave at infinity (i.e. the greater $\beta$ becomes), the slightly better estimates we have as lower bounds.

Example 2.4. Let us consider a solution $u(x)$ of the equation (2.2) for $x$ $\in \Omega:=\left\{x|| x \mid>R_{0}\right\}$ such that $\operatorname{supp}[u]$ is not a compact set in $\bar{\Omega}$.

We assume that there exists some constant $\beta \geq 0$ such that

$h(x)$ is a homogeneous function of degree $0>\alpha>-2$ and there exist some constants $0<d_{1}<1$ and $\delta \geq 0$ such that for any $w(x) \in C_{0}^{\infty}(\Omega)$ we have

$$
\int_{\Omega}(h)_{-}|w|^{2} d x \leq \int_{\Omega}\left\{d_{1}|\nabla w|^{2}+\text { Const } r^{\delta}|w|^{2}\right\} d x ;
$$

(here we assume neither $h(x)>0$ nor $h(x) \in L_{l o c}^{\infty}(\Omega)$,)

$V_{1}(x) \in C^{1}(\bar{\Omega})$ is a real-valued function satisfying

$$
V_{1}(x)=0(1), \partial_{r} V_{1}(x)=0\left(r^{-1}\right) \text { as } r \rightarrow \infty ;
$$

$V_{2}(x) \in C^{0}(\bar{\Omega})$ is a complex-valued function satisfying

$$
V_{2}(x)=\mathrm{o}\left(r^{-1}(\log r)^{-\beta / 2}\right) \text { as } r \rightarrow \infty ;
$$

$Q(r) \in C^{1}(\bar{\Omega})$ is a real-valued function satisfying

$$
Q(r)=\mathrm{o}\left((\log r)^{-\beta}\right) \text { as } r \rightarrow \infty ;
$$

$b_{j}(x) \in C^{1}(\bar{\Omega})$ is a real-valued function satisfying

$$
|B(x) x|=0(1) \text { as } r \rightarrow \infty ;
$$

$\lambda$ is a constant satisfying $\lambda>0$.

Then we have

$$
\left\{\begin{array}{lr}
\lim _{R \rightarrow \infty} R^{\delta-(\alpha / 2)} \exp \left\{\varepsilon(\log R)^{1-\beta}\right\} \int_{R<|x|<R+1}|u|^{2} d x=\infty & \text { for any } \varepsilon>0 \text { if } 0 \leq \beta<1 ; \\
\lim _{R \rightarrow \infty} R^{\delta-(\alpha / 2)}(\log R)^{\varepsilon} \int_{R<|x|<R+1}|u|^{2} d x=\infty & \text { for any } \varepsilon>0 \text { if } \beta=1 ; \\
\liminf _{R \rightarrow \infty} R^{\delta-(\alpha / 2)} \int_{R<|x|<R+1}|u|^{2} d x>0 & \text { if } \beta>1 ; \\
u(x) \notin L^{2}\left(|x|>R_{0}\right) & \text { if } 2+\alpha>2 \delta, \beta \geq 0 \text { or } 2+\alpha=2 \delta, \beta \geq 1 .
\end{array}\right.
$$


Proof of Example 2.4. Let $q_{1}(x)=-h(x)+V_{1}(x)-\lambda$ and $q_{2}(x)=V_{2}(x)$ $+r^{-1} Q^{\prime}(r)$. Let $\varepsilon>0$ be arbitrary, and let

$$
\begin{aligned}
& \psi_{i}(r)=(\log r)^{-(\beta / 2)}(i=1,2), \\
& \sigma_{i}(r)=2 \varepsilon(\log r)^{-\beta}-4 Q(r)(i=1,2), \\
& \eta_{i}(r)=-\alpha(i=1,2), \\
& \tau(r) \equiv \tau_{0} \text { be a constant satisfying } 0<\tau_{0}<-\alpha, \\
& Q_{0}(r)=Q(r), \\
& a_{1}>1, a_{2}>0 \text { be arbitrary constants. }
\end{aligned}
$$

By $Q(r)=\mathrm{o}\left((\log r)^{-\beta}\right)$, there exists some $R_{2} \geq R_{0}$ such that for any $r \geq R_{2}$ we have $|Q(r)|<4^{-1} \varepsilon(\log r)^{-\beta}$ and $\log r \geq 1$. Then for any $r \geq R_{2}$

$$
\varepsilon(\log r)^{-\beta}<\sigma_{i}(r)<3 \varepsilon(\log r)^{-\beta} \leq 3 \varepsilon
$$

So we can easily verify that the conditions (E.1)-(E.6) hold. We have for $r \geq$ $R_{2}$

$$
\psi_{2}^{2} \sigma_{2}^{-1} \exp \left(\int_{R_{0}}^{r} \frac{\tau(t)-\eta_{2}(t)}{t} d t\right) \leq \text { Const } r^{\tau_{0}+\alpha}
$$

which leads us to (E.7). Now we check (F.2). Note that $Q_{i}(r)=4^{-1}\left(\eta_{i}-\sigma_{i}\right)=$ $Q(r)-4^{-1}\left\{2 \varepsilon(\log r)^{-\beta}+\alpha\right\}$. We have

$$
\begin{aligned}
\phi_{i}^{2} \sigma_{i}^{-1}\left\{r \partial_{r} q_{1}+\eta_{i} q_{1}+a_{i} \sigma_{i}^{-1}\left|r q_{2}-Q_{i}^{\prime}\right|^{2}+\left(2-\eta_{i}\right)^{-1}|B x|^{2}\right\} \\
=\psi_{i}^{2} \sigma_{i}^{-1}\left\{\alpha \lambda+\left(r \partial_{r} V_{1}-\alpha V_{1}\right)\right. \\
\left.\quad+a_{i} \sigma_{i}^{-1}\left|r V_{2}-2^{-1} \varepsilon \beta r^{-1}(\log r)^{-1-\beta}\right|^{2}+(2+\alpha)^{-1}|B x|^{2}\right\} .
\end{aligned}
$$

Therefore, by $\psi_{i}^{2} \sigma_{i}^{-1} \geq(3 \varepsilon)^{-1}$, we have (F.2). (1.1) is satisfied by $\delta$ given in the condition for $h(x)$. Since $\sigma_{1}(r)+\eta_{1}(r) \leq 3 \varepsilon(\log r)^{-\beta}-\alpha$ for $r \geq R_{2}$, we have for any $R \geq R_{2}$

$$
\begin{aligned}
\Phi_{1}(R) & =\exp \left(\int_{R_{0}}^{R} \frac{\sigma_{1}(t)+\eta_{1}(t)}{2 t} d t\right) \\
& \leq \text { Const } \exp \left(\int_{R_{2}}^{R} \frac{3 \varepsilon(\log t)^{-\beta}-\alpha}{2 t} d t\right) \\
& = \begin{cases}\text { Const } R^{-(\alpha / 2)} \exp \left\{\frac{3 \varepsilon}{2(1-\beta)}(\log R)^{1-\beta}\right\} & \text { if } \beta \neq 1, \\
\text { Const } R^{-(\alpha / 2)}(\log R)^{(3 \varepsilon) / 2} & \text { if } \beta=1 .\end{cases}
\end{aligned}
$$

Using the arbitrariness of $\varepsilon>0$, we can replace $(3 / 2) \varepsilon(1-\beta)^{-1}$ (in case $0 \leq \beta<$ 1 ) or $(3 \varepsilon / 2)$ (in case $\beta=1$ ) with $\varepsilon$. Thus by Corollary 1.3 we have the first three assertions, from which we can easily see the last assertion.

Remark 2.5. The following atomic type many body potential 


$$
-h(x)=-\sum_{j=1}^{N} \frac{z_{j}}{\left|x^{(j)}\right|}+\sum_{1 \leq j<k \leq N} \frac{z_{j k}}{\left|x^{(j)}-x^{(k)}\right|}
$$

is a homogeneous function of degree $\alpha=-1$, do not have a constant sign, is not of $L_{\text {loc }}^{\infty}(\Omega)$ but satisfies (1.1) with $\delta=0$ and Assumptions (C.2) and (C.3), (see, e.g. Eastham-Kalf [3, pp. 242-249],) where $z_{j}$ and $z_{j k}$ are positive constants, $n$ $=3 N, x={ }^{t}\left(x^{(1)}, \cdots, x^{(N)}\right) \in \boldsymbol{R}^{n}$ and $x^{(j)} \in \boldsymbol{R}^{3}$. Thus we can apply Example 2.4 to this $h(x)$.

\section{§3. Integrations by Parts}

In this section we put $\Omega:=\left\{x|| x \mid>R_{0}\right\}$. We assume in this section the assumptions concerning the regularities of $b_{j}(x), q_{1}(x), q_{2}(x), \sigma_{i}(r)$ and $\eta_{i}(r)$ and do not assume any estimates. Namely we only assume Assumptions (B), (C), (D) and that $\phi_{i}(r), \sigma_{i}(r), \eta_{i}(r) \in C^{1}\left[R_{0}, \infty\right)$ are real-valued.

Definition 3.1. Let $u \in H_{l o c}^{2}(\Omega)$ satisfy the equation (0.1). For a real valued function $\rho(r) \in C^{2}\left[R_{0}, \infty\right)$, we put

$$
v=v(x ; \rho)=e^{\rho(|x|)} u(x) .
$$

Lemma 3.2. The function $v \in H_{\text {loc }}^{2}(\Omega)$ defined above satisfies the equation

$$
-\langle D, D v\rangle+2 \rho^{\prime}(r) D_{r} v+q_{\rho}(x) v=0
$$

where we put

$$
q_{\rho}(x):=q_{1}(x)+q_{2}(x)+\rho^{\prime \prime}(r)+(n-1) r^{-1} \rho^{\prime}(r)-\rho^{\prime}(r)^{2} .
$$

Proof. Since $u=e^{-\rho} v$, we have

$$
\begin{aligned}
\langle D, D u\rangle & =\left\langle D, e^{-\rho}(D v-(D \rho) v)\right\rangle \\
& =e^{-\rho}\{\langle D,(D v-(D \rho) v)\rangle-\langle D \rho,(D v-(D \rho) v)\rangle\} \\
& =e^{-\rho}\left\{\langle D, D v\rangle-\left\langle D, \rho^{\prime} v \bar{x}\right\rangle-\rho^{\prime} D_{r} v+\rho^{\prime 2} v\right\} \\
& =e^{-\rho}\left\{\langle D, D v\rangle-2 \rho^{\prime} D_{r} v-\left[\rho^{\prime \prime}+(n-1) r^{-1} \rho^{\prime}-\rho^{\prime 2}\right] v\right\},
\end{aligned}
$$

which with the equation (0.1) shows the present lemma.

Lemma 3.3. Let $v$ be as in Definition 3.1. Let $\Phi(r), f(r)$ and $g(r)$ be real-valued functions of class $C^{1}\left[R_{0}, \infty\right)$. Then for $R_{0}<s<t$ we have

$$
\left(\int_{|x|=t}-\int_{|x|=s}\right) \Phi(r)\left[2 f(r)\left|D_{r} v\right|^{2}-f(r)|D v|^{2}+g(r) \operatorname{Re}\left[\bar{v} D_{r} v\right]\right] d S
$$




$$
\begin{aligned}
(3.3)=\int_{s<|x|<t} & {\left[\left\{\Phi g+(\Phi f)^{\prime}-(n-1) r^{-1} \Phi f+4 \rho^{\prime} \Phi f\right\}\left|D_{r} v\right|^{2}\right.} \\
& +\left\{\Phi g-(\Phi f)^{\prime}-(n-3) r^{-1} \Phi f\right\}\left(|D v|^{2}-\left|D_{r} v\right|^{2}\right) \\
& +\operatorname{Re}\left[\left\{(\Phi g)^{\prime}+2 \Phi\left(\rho^{\prime} g+f \overline{q \rho}\right)\right\} \bar{v} D_{r} v\right] \\
& \left.-2 \Phi f \operatorname{Re}[\sqrt{-1}\langle B \bar{x}, D v\rangle v]+\Phi g \operatorname{Re}\left[q_{\rho}\right]|v|^{2}\right] d x
\end{aligned}
$$

Proof. Multiplying the both side of (3.1) by $\Phi\left\{2 f \overline{D_{r} v}+g \bar{v}\right\}$ and integrating on $s<|x|<t$, we have

$$
\begin{aligned}
& -2 \int_{s<|x|<t}\left[\Phi(r) f(r)\langle D, D v\rangle \overline{D_{r} v}\right] d x \\
& -\int_{s<|x|<t}[\Phi(r) g(r)\langle D, D v\rangle \bar{v}] d x \\
& +\int_{s<|x|<t}\left[\Phi(r)\left\{2 \rho^{\prime} D_{r} v+q_{\rho} v\right\}\left\{2 f(r) \overline{D_{r} v}+g(r) \bar{v}\right\}\right] d x=0 .
\end{aligned}
$$

Now, noting (B) the first integral of (3.4) is

$$
\begin{aligned}
& -2 \int_{s<|x|<t}\left[\Phi f\langle D, D v\rangle \overline{D_{r} v}\right] d x \\
= & -2\left(\int_{|x|=t}-\int_{|x|=s}\right)\left[\Phi f\left|D_{r} v\right|^{2}\right] d S \\
& +2 \int_{s<|x|<t}\left[(\Phi f)^{\prime}\left|D_{r} v\right|^{2}+r^{-1} \Phi f\left(|D v|^{2}-\left|D_{r} v\right|^{2}\right)\right] d x \\
& +2 \int_{s<|x|<t}\left[\Phi f \sum_{j, k=1}^{n} D_{j} v \cdot \widehat{x}_{k} \overline{D_{j} D_{k} v}\right] d x .
\end{aligned}
$$

By

$$
D_{j} D_{k} v-D_{k} D_{j} v=\sqrt{-1} B_{j k}(x) v,
$$

the last integral in the right side of (3.5) is

$$
\begin{aligned}
& \int_{s<|x|<t}\left[\Phi f \sum_{j, k=1}^{n} D_{j} v \cdot \bar{x}_{k} \overline{D_{j} D_{k} v}\right] d x \\
= & \int_{s<|x|<t}\left[\Phi f \sum_{j, k=1}^{n} D_{j} v \cdot \widehat{x}_{k} \overline{D_{k} D_{j} v}\right] d x \\
& -\sqrt{-1} \int_{s<|x|<t}[\Phi f\langle B \hat{x}, D v\rangle \bar{v}] d x .
\end{aligned}
$$

The first term in the right side of (3.7) is

$$
\begin{aligned}
& \int_{s<|x|<t}\left[\Phi f \sum_{j, k=1}^{n} D_{j} v \cdot \widehat{x}_{k} \overline{D_{k} D_{j} v}\right] d x \\
& =\left(\int_{|x|=t}-\int_{|x|=s}\right)\left[\Phi f|D v|^{2}\right] d S \\
& \quad-\int_{s<|x|<t}\left[\left\{(\Phi f)^{\prime}+(n-1) r^{-1} \Phi f\right\}|D v|^{2}\right] d x
\end{aligned}
$$




$$
-\int_{s<|x|<t}\left[\Phi f \sum_{j, k=1}^{n} D_{k} D_{j} v \cdot \bar{x}_{k} \overline{D_{j} v}\right] d x
$$

Using again (3.6), the last integral in the above is

$$
\begin{aligned}
& \int_{s<|x|<t}\left[\Phi f \sum_{j, k=1}^{n} D_{k} D_{j} v \cdot \widehat{x}_{k} \overline{D_{j} v}\right] d x \\
& =\int_{s<|x|<t}\left[\Phi f \sum_{j, k=1}^{n} D_{j} D_{k} v \cdot \widehat{x}_{k} \overline{D_{j} v}\right] d x \\
& -\sqrt{-1} \int_{s<|x|<t}[\Phi f\langle B \hat{x}, \overline{D v}\rangle v] d x .
\end{aligned}
$$

Since the first term in the right side of the above formula is complex conjugate to the left side of (3.7), we have

$$
\begin{aligned}
& 2 \operatorname{Re} \int_{s<|x|<t}\left[\Phi f \sum_{j, k=1}^{n} D_{j} v \cdot \widehat{x}_{k} \overline{D_{j} D_{k} v}\right] d x \\
= & \left(\int_{|x|=t}-\int_{|x|=s}\left[\Phi f|D v|^{2}\right] d S\right. \\
& -\int_{s<|x|<t}\left[\left\{(\Phi f)^{\prime}+(n-1) r^{-1} \Phi f\right\}|D v|^{2}\right] d x \\
& -2 \operatorname{Re} \int_{s<|x|<t}[\Phi f \sqrt{-1}\langle B \bar{x}, D v\rangle \bar{v}] d x .
\end{aligned}
$$

Next the second integral of (3.4) is

$$
-\int_{s<|x|<t}[\Phi g\langle D, D v\rangle \bar{v}] d x
$$

(3.9) $=-\left(\int_{|x|=t}-\int_{|x|=s}\right)\left[\Phi g \bar{v} D_{r} v\right] d S+\int_{s<|x|<t}\left[(\Phi g)^{\prime} \bar{v} D_{r} v+\Phi g|D v|^{2}\right] d x$.

Taking the real part of (3.4) and using (3.5), (3.8) and (3.9), we have the assertion.

Lemma 3.4. Let $\psi(x)$ be a function satisfying the conditions $\psi(x)$ is a real-valued function,

for any $w(x) \in H_{\text {loc }}^{1}(\Omega)$ we have $\phi(x)|w(x)|^{2} \in L_{l o c}^{1}(\Omega)$, for any $w(x) \in H_{\text {loc }}^{1}(\Omega)$ we have $\partial_{r} \psi(x) \cdot|w(x)|^{2} \in L_{\text {loc }}^{1}(\Omega)$.

Then for any $w \in H_{\text {loc }}^{2}(\Omega)$ and for $R_{0}<s<t$, it holds that

$$
\begin{aligned}
& \left(\int_{|x|=t}-\int_{|x|=s}\right)\left[\psi(x)|w|^{2}\right] d S \\
& \quad=\int_{s<|x|<t}\left[2 \psi(x) \operatorname{Re}\left[\bar{w} D_{r} w\right]+\left\{\partial_{r} \psi(x)+(n-1) r^{-1} \psi(x)\right\}|w|^{2}\right] d x .
\end{aligned}
$$

Proof. If $\psi$ and $w$ were $C^{\infty}$, this would follow easily by integration by parts. A rigorous proof of this lemma will be obtained from Uchiyama [12, Lemma 4.1]. 
Lemma 3.5. Let $\rho(r) \in C^{2}\left[R_{0}, \infty\right), f(r) \in C^{1}\left[R_{0}, \infty\right), g(r) \in C^{1}\left[R_{0}, \infty\right)$ and $\gamma(r) \in C^{1}\left[R_{0}, \infty\right)$ be real valued functions. Let $\widetilde{\phi}(x)$ satisfy the Assumptions given in Lemma 3.4 with $\psi$ replaced with $\widetilde{\phi}$. We put

$$
\Phi(r)=\exp \left[\int_{R_{0}}^{r} \gamma(t) d t\right]
$$

Then for any $t>s>R_{0}$ we have

$$
\begin{aligned}
& \left(\int_{|x|=t}-\int_{|x|=s}\right) \Phi(r)\left[2 f(r)\left|D_{r} v\right|^{2}-f|D v|^{2}\right. \\
& \left.+g(r) \operatorname{Re}\left[\bar{v} D_{r} v\right]+f \widetilde{\phi}(x)|v|^{2}\right] d S \\
& =\int_{s<|x|<t} \Phi\left[\left\{g+\gamma f-(n-1) r^{-1} f+f^{\prime}+4 \rho^{\prime} f\right\}\left|D_{r} v\right|^{2}\right. \\
& +\left\{g-\gamma f-(n-3) r^{-1} f-f^{\prime}\right\}\left\{|D v|^{2}-\left|D_{r} v\right|^{2}\right\} \\
& +\operatorname{Re}\left[\left\{\gamma g+2\left(\rho^{\prime} g+f \overline{q_{\rho}}+f \widetilde{\phi}\right)+g^{\prime}\right\} \bar{v} D_{r} v\right] \\
& -2 f \operatorname{Re}\left[\left\langle\sqrt{-1} B \hat{x}, D v-\bar{x} D_{r} v\right\rangle \bar{v}\right] \\
& \left.+\left\{g \operatorname{Re}\left[q_{\rho}\right]+f \partial_{r} \widetilde{\phi}+\widetilde{\phi}\left(\gamma f+f^{\prime}+(n-1) r^{-1} f\right)\right\}|v|^{2}\right] d x \text {. }
\end{aligned}
$$

Proof. Let $\phi=\Phi f \widetilde{\phi}$. Then, $\psi$ satisfies the conditions given in Lemma 3. 4. Add (3.3) and (3.10) with $\psi=\Phi f \widetilde{\phi}$ and $w=v$. Note that

$$
\langle B \hat{x}, \hat{x}\rangle=0,
$$

where we used the fact that $B$ is real and skew-symmetric. Then we have (3.11).

Definition 3.6. Let $\sigma_{i}(r) \in C^{1}\left[R_{0}, \infty\right)$ and $\eta_{i}(r) \in C^{1}\left[R_{0}, \infty\right)$ be the functions given in our Assumptions. We put

$$
\begin{aligned}
& \gamma_{i}(r)=\frac{\sigma_{i}(r)+\eta_{i}(r)}{2 r} \\
& g_{i}(r)=\frac{\sigma_{i}(r)-\eta_{i}(r)}{2 r}+(n-1) r^{-1}=-2 r^{-1} Q_{i}(r)+(n-1) r^{-1} \\
& \Phi_{i}(r)=\exp \left(\int_{R_{0}}^{r} \gamma_{i}(t) d t\right)
\end{aligned}
$$

where

$$
Q_{i}(r)=4^{-1}\left(\eta_{i}(r)-\sigma_{i}(r)\right)
$$

By Assumptions $g_{i}(r), \Phi_{i}(r)$ and $Q_{i}(r)$ are real-valued functions of class $C^{1}\left[R_{0}, \infty\right)$.

Definition 3.7. For real-valued functions $\rho(r) \in C^{2}\left[R_{0}, \infty\right)$ and $\phi(r) \in$ $C^{1}\left[R_{0}, \infty\right)$, and a complex-valued function $w(x) \in H_{\text {loc }}^{2}(\Omega)$, we put 
(1) $\quad F_{i}(t ; \rho, \phi ; w)=\int_{|x|=t} \Phi_{i}(r) J_{i}(x ; \rho, \phi ; w) d S \quad(i=1,2)$,

(2) $\quad J_{i}(x ; \rho, \phi ; w)=2\left|D_{r} w\right|^{2}-|D w|^{2}+g_{2}(r) \operatorname{Re}\left[\bar{w} D_{r} w\right]$

$$
+\left\{-q_{1}(x)+\rho^{\prime 2}+4^{-1} g_{i}(r)^{2}-\phi(r)\right\}|w|^{2},
$$

(3) $\quad G_{i}(x ; \rho, \phi ; w)=\sum_{j=0}^{2} G_{i}^{(j)}(x ; \rho, \phi ; w)$,

(4) $G_{i}^{(2)}(x ; \rho, \phi ; w)$

$$
=\left\{\sigma_{i}(r)+4 r \rho^{\prime}(r)\right\}\left|D_{r} w\right|^{2}+\left\{2-\eta_{i}(r)\right\}\left\{|D w|^{2}-\left|D_{r} w\right|^{2}\right\},
$$

(5) $G_{i}^{(1)}(x ; \rho, \phi ; w)=2 \operatorname{Re}\left[\left\{r \overline{q_{2}(x)}-Q_{i}^{\prime}(r)\right\} \bar{w} D_{r} w\right]$

$$
+r\left\{\gamma_{i}(r) g_{i}+2 r^{-2} Q_{i}-(n-1) r^{-2}+2^{-1} g_{i}^{2}\right\} \operatorname{Re}\left[\bar{w} D_{r} w\right]
$$$$
-2 \operatorname{Re}\left[\left\langle\sqrt{-1} B x, D w-\bar{x} D_{r} w\right\rangle \bar{w}\right]
$$$$
+2 r\left\{\rho^{\prime \prime}+\rho^{\prime} g_{i}+(n-1) r^{-1} \rho^{\prime}-\phi\right\} \operatorname{Re}\left[\bar{w} D_{r} w\right] \text {, }
$$

(6) $G_{i}^{(0)}(x ; \rho, \phi ; w)=\left[-\left\{r \partial_{r} q_{1}+\eta_{i} q_{1}\right\}+g_{i}\left\{r \operatorname{Re}\left[q_{2}\right]-Q_{i}^{\prime}\right\}\right.$

$$
\begin{aligned}
& +4^{-1} r g_{i}^{2}\left\{\gamma_{i}+(n-1) r^{-1}\right\}+r^{-1} g_{i} Q_{i}-2^{-1}(n-1) r^{-1} g_{i} \\
& +\left\{r\left(\rho^{\prime 2}\right)^{\prime}+\eta_{i} \rho^{\prime 2}\right\}+r g_{i}\left\{\rho^{\prime \prime}+(n-1) r^{-1} \rho^{\prime}\right\} \\
& \left.-r\left\{\phi\left(\gamma_{i}+(n-1) r^{-1}\right)+\phi^{\prime}\right\}\right]|w|^{2} .
\end{aligned}
$$

Note that the superscript $j$ of $G_{i}^{(j)}$ corresponds to the order of $D w$.

Lemma 3.8. It holds that

$$
\begin{aligned}
& F_{i}(t ; \rho, \phi ; v(\cdot, \rho))-F_{i}(s ; \rho, \phi ; v(\cdot, \rho)) \\
& \quad=\int_{s<|x|<t} \Phi_{i}(r) r^{-1} G_{i}(x ; \rho, \phi ; v(\cdot, \rho)) d x
\end{aligned}
$$

for $R_{0}<s<t$.

Proof. Let

$$
\begin{aligned}
& f(r)=1, \gamma(r)=\gamma_{i}(r), g(r)=g_{i}(r), \\
& \widetilde{\phi}(x)=-q_{1}(x)+\rho^{\prime}(r)^{2}+4^{-1} g_{i}(r)^{2}-\phi(r) .
\end{aligned}
$$

By (C.1), (C.2) and (C.3), we can apply (3.11). Then it is easy to see that the left side of (3.11) reduces to the left side of (3.12).

One can also show that the right side of (3.11) reduces to the right side of (3.12) by using the identities

$$
\begin{aligned}
& g+\gamma-(n-1) r^{-1}=r^{-1} \sigma_{i}(r), \\
& g-\gamma-(n-1) r^{-1}=-r^{-1} \eta_{i}(r), \\
& \overline{q_{\rho}}+\widetilde{\phi}=\overline{q_{2}}+4^{-1} g_{i}^{2}-\phi+\rho^{\prime \prime}+(n-1) r^{-1} \rho^{\prime}, \\
& 2 \overline{q_{2}}+g^{\prime}=2\left\{\overline{q_{2}}-r^{-1} Q_{i}^{\prime}\right\}+2 r^{-2} Q_{i}-(n-1) r^{-2},
\end{aligned}
$$




$$
4^{-1}\left(g^{2}\right)^{\prime}=\left\{-r^{-1} Q_{i}^{\prime}+r^{-2} Q_{i}-2^{-1}(n-1) r^{-2}\right\} g_{i},
$$

where in the third identity we used the definition of $q_{\rho}(x)$ given by (3.2) in Lemma 3.2.

\section{§4. Fundamental Estimates}

In this section we will not use (E.5)-(E.7) and (G).

Lemma 4.1. Asssume (E.1)-(E.4) and (F.1), (F.2). Then there exist positive constants $C_{2}, C_{3}$ and $R_{3} \geq R_{0}$ such that for any $|x| \geq R_{3}$ and for any $w \in H_{\text {loc }}^{2}(\Omega)$ we have

(1) $G_{1}(x ; 0,0, w) \geq 0$,

(2) $G_{2}(x ; 0,0 ; w) \geq-C_{2} \sigma_{2}(x)\left|D_{r} w\right|^{2}+C_{3} \psi_{2}(x)^{-2} \sigma_{2}(x)|w|^{2}$.

Proof. Let $\varepsilon_{1}, \varepsilon_{2}$ and $\varepsilon_{3}$ be positive constants, which will be determined later. Then the following estimates hold for large $r$, say $r \geq R$, where $R$ may depend on $\varepsilon$ 's.

Firstly let us show

$$
\begin{aligned}
& G_{i}^{(1)}(x ; 0,0 ; w) \\
& \geq-\left(\varepsilon_{1}+\varepsilon_{2}\right) \sigma_{i}\left|D_{r} w\right|^{2}-\left(2-\eta_{i}\right)\left\{|D w|^{2}-\left|D_{r} w\right|^{2}\right\} \\
& -\left[\varepsilon_{1}^{-1} \sigma_{i}^{-1}\left|r q_{2}(x)-Q_{i}^{\prime}(r)\right|^{2}+\left(2-\eta_{i}\right)^{-1}|B x|^{2}+\varepsilon_{2} \psi_{i}^{-2} \sigma_{i}\right]|w|^{2} \text {. }
\end{aligned}
$$

First we note $g_{i}=\mathrm{O}\left(r^{-1}\right), \quad \gamma_{i}=\mathrm{O}\left(r^{-1}\right), Q_{i}=\mathrm{O}(1)$ by (E.1), (E.2) and Definition 3.6. By (E.4), we have

$$
\gamma_{i} g_{i}+2 r^{-2} Q_{i}-(n-1) r^{-2}+2^{-1} g_{i}^{2}=\mathrm{O}\left(r^{-2}\right)=\mathrm{o}(1) \cdot r^{-1} \psi_{i}^{-1} \sigma_{i}
$$

Noting

$$
\left|D w-\hat{x} D_{r} w\right|^{2}=|D w|^{2}-\left|D_{r} w\right|^{2}
$$

we have

$$
\begin{aligned}
& 2 \operatorname{Re}\left[\left\{r \overline{q_{2}}-Q_{i}^{\prime}(r)\right\} \bar{w} D_{r} w\right] \\
& \quad \geq-\varepsilon_{1} \sigma_{i}\left|D_{r} w\right|^{2}-\varepsilon_{1}^{-1} \sigma_{i}^{-1}\left|r q_{2}-Q_{i}^{\prime}(r)\right|^{2}|w|^{2}, \\
& r\left\{\gamma_{i} g_{i}+2 r^{-2} Q_{i}-(n-1) r^{-2}+2^{-1} g_{i}^{2}\right\} \operatorname{Re}\left[\bar{w} D_{r} w\right] \\
& \quad=0(1) \cdot\left\{\sigma_{i}\left|D_{r} w\right|^{2}+\psi_{i}^{-2} \sigma_{i}|w|^{2}\right\} \\
& \quad \geq-\varepsilon_{2}\left\{\sigma_{i}\left|D_{r} w\right|^{2}+\psi_{i}^{-2} \sigma_{i}|w|^{2}\right\}, \\
& -2 \operatorname{Re}\left[\left\langle\sqrt{-1} B x, D w-\bar{x} D_{r} w\right\rangle \bar{w}\right] \\
& \quad \geq-\left(2-\eta_{i}\right)\left\{|D w|^{2}-\left|D_{r} w\right|^{2}\right\}-\left(2-\eta_{i}\right)^{-1}|B x|^{2}|w|^{2} .
\end{aligned}
$$

Collecting the above estimates, we have (4.1). 
If $B(x) \equiv 0$, we need not use the existence $\left(2-\eta_{i}\right)^{-1}$. Then in this case we can replace (E.2) with (E.2)' in Remark 1.4.

Lastly let us show

$$
\begin{aligned}
G_{i}^{(0)}(x & ; 0,0 ; w) \\
\geq- & {\left[\left\{r \partial_{r} q_{1}(x)+\eta_{i}(r) q_{1}(x)\right\}\right.} \\
& \left.+\left(a_{i}-\varepsilon_{1}^{-1}\right) \sigma_{i}^{-1}\left|r q_{2}(x)-Q_{i}^{\prime}(r)\right|^{2}+\varepsilon_{3} \psi_{i}^{-2} \sigma_{i}\right]|w|^{2},
\end{aligned}
$$

if $a_{i}^{-1}<\varepsilon_{1}$.

Note that $g_{i}=\mathrm{O}\left(r^{-1}\right)$ and $r^{-2}=\mathrm{o}(1) \psi^{-2} \sigma_{i}$ by (E.1) and (E.4). Then, assuming $a_{i}-\varepsilon_{1}^{-1}>0$, we can see that

$$
\begin{aligned}
& g_{i}\left\{r \operatorname{Re}\left[q_{2}\right]-Q_{i}^{\prime}\right\} \geq-\left\{\left(a_{i}-\varepsilon_{1}^{-1}\right) \sigma_{i}^{-1}\left|r \operatorname{Re}\left[q_{2}\right]-Q_{i}^{\prime}\right|^{2}+2^{-1} \varepsilon_{3} \psi_{i}^{-2} \sigma_{i}\right\}, \\
& 4^{-1} r g_{i}^{2}\left\{\gamma_{i}+(n-1) r^{-1}\right\}+r^{-1} g_{i} Q_{i}-2^{-1}(n-1) r^{-1} g_{i} \\
& \quad=\mathrm{O}\left(r^{-2}\right) \geq-2^{-1} \varepsilon_{3} \psi_{i}^{-2} \sigma_{i},
\end{aligned}
$$

which and Definition 3.7 (6) show (4.2).

Adding Definition 3.7 (4), (4.1) and (4.2), we have

$$
\begin{aligned}
& G_{i}(x ; 0,0 ; w) \\
& \quad \geq\left(1-\varepsilon_{1}-\varepsilon_{2}\right) \sigma_{i}\left|D_{r} w\right|^{2} \\
& \quad-\left[r \partial_{r} q_{1}+\eta_{i} q_{1}+a_{i} \sigma_{i}^{-1}\left|r q_{2}-Q_{i}^{\prime}\right|^{2}+\left(2-\eta_{i}\right)^{-1}|B x|^{2}\right]|w|^{2} \\
& \quad-\left(\varepsilon_{2}+\varepsilon_{3}\right) \psi_{i}^{-2} \sigma_{i}|w|^{2},
\end{aligned}
$$

if $a_{i}^{-1}<\varepsilon_{1}$.

Let us choose $\varepsilon$ 's appropriately corresponding to the cases $i=1$ and $i=2$. By (F.2) there exist some $R_{3} \geq R_{0}$ and $C_{3}>0$ such that for any $r \geq R_{3}$ we have

$$
\begin{aligned}
& -\left[r \partial_{r} q_{1}+\eta_{i} q_{1}+a_{i} \sigma_{i}^{-1}\left|r q_{2}-Q_{i}^{\prime}\right|^{2}+\left(2-\eta_{i}\right)^{-1}|B x|^{2}\right] \\
& \geq 2 C_{3} \psi_{i}^{-2} \sigma_{i} .
\end{aligned}
$$

In case $i=2$, by (F.1) we can choose $\varepsilon_{1}$ so large and $\varepsilon_{2}$ and $\varepsilon_{3}$ so small that

$$
\begin{aligned}
& \varepsilon_{1}>a_{2}^{-1}, \\
& -\left(\varepsilon_{2}+\varepsilon_{3}\right) \geq-C_{3},
\end{aligned}
$$

which leads us to the assertion (2).

In case $i=1$, by (F.1) we can choose $\varepsilon_{1}$ such that

$$
1>\varepsilon_{1}>a_{1}^{-1} \text {. }
$$

If we choose $\varepsilon_{2}$ and $\varepsilon_{3}$ so small, we have

$$
\begin{aligned}
& 1-\varepsilon_{1}-\varepsilon_{2}>0, \\
& -\left(\varepsilon_{2}+\varepsilon_{3}\right) \geq-C_{3},
\end{aligned}
$$

which lead us to the assertion (1). 
In $§ \S 5-7$ we will not use the assumption (E.2) itself but only use the validity of Lemma 4.1. Thus if Theorems 1.1 and 1.2 are proved, then the assertion of Remark 1.4 also hold.

\section{\$5. Monotonicity and Positivity of $F_{i}$}

In this section we assume (B)-(E) and the validities of Lemmas 3.8 and 4.1.

Proposition 5.1. Suppose that there exists $R_{*}>R_{3}$ such that

$$
F_{1}\left(R_{*} ; 0,0 ; v(\cdot ; 0)\right)=F_{1}\left(R_{*} ; 0,0 ; u\right)>0
$$

then Theorem 1.1 holds.

Proof. By Lemma 3.8 and Lemma $4.1(1), F_{1}(t ; 0,0 ; u)$ is non-decreasing in $t \geq R_{3}$ so that we have for any $t \geq R_{*}$

$$
\begin{aligned}
0< & F_{1}\left(R_{*} ; 0,0 ; u\right) \\
\leq & F_{1}(t ; 0,0 ; u) \\
= & \int_{|x|=t} \Phi_{1}(r)\left[\left|D_{r} u\right|^{2}+\left\{\left|D_{r} u\right|^{2}-|D u|^{2}\right\}\right. \\
& \left.+g_{1}(r) \operatorname{Re}\left[\bar{u} D_{r} u\right]+\left\{-\left(q_{1}\right)_{+}+\left(q_{1}\right)_{-}+4^{-1} g_{1}^{2}\right\}|u|^{2}\right] d S \\
\leq & \int_{|x|=t} \Phi_{1}(r)\left[\left|D_{r} u\right|^{2}+g_{1} \operatorname{Re}\left[\bar{u} D_{r} u\right]+\left\{\left(q_{1}\right)_{-}+4^{-1} g_{1}^{2}\right\}|u|^{2}\right] d S \\
\leq & \int_{|x|=t} \Phi_{1}(r)\left[2\left|D_{r} u\right|^{2}+\left\{\left(q_{1}\right)_{-}+2^{-1} g_{1}^{2}\right\}|u|^{2}\right] d S \\
\leq & \text { Const } \int_{|x|=t} \Phi_{1}(r)\left[\left|D_{r} u\right|^{2}+\left\{\left(q_{1}\right)_{-}+r^{-2}\right\}|u|^{2}\right] d S,
\end{aligned}
$$

which shows Theorem 1.1.

Lemma 5.2. Assume (E.1), (E.3) and (E.4) with $i=2$. Then there exists a function $\xi(r) \in C^{1}\left[R_{0}, \infty\right)$ such that

(i) $\xi(r)>0$ for any $r \geq R_{0}$,

(ii) $\lim _{r \rightarrow \infty} \xi(r)=\infty$,

(iii) $\lim _{r \rightarrow \infty} r^{-1} \psi_{2}(r) \sigma_{2}(r)^{-1} \xi(r)=0$,

(iv) $\lim _{r \rightarrow \infty} r^{-1} \psi_{2}(r)^{2} \sigma_{2}(r)^{-1} \xi^{\prime}(r)=0$.

Proof. Note (E.4). We put $\varphi_{0}(t)=C_{4} \sup _{r \geq t} r^{-1} \psi_{2}(r) \sigma_{2}(r)^{-1}$, where $C_{4}>0$ is chosen to satisfy $\varphi_{0}\left(R_{0}\right)^{-1}>2$. Then $\varphi_{0}(t)^{-1}$ is non-decreasing and positive in 
$t \in\left[R_{0}, \infty\right)$ by (E.1) and (E.3), and tends to $\infty$ as $t$ goes to $\infty$ by (E.4). We define $\left\{r_{j}\right\}_{j=1,2, \cdots}$ inductively as follows :

$$
r_{1}=R_{0} ; r_{j+1}>r_{j}+1 \text { and } \varphi_{0}\left(r_{j+1}\right)^{-1} \geq j+2 .
$$

Let $\xi_{0}(t) \in C^{\infty}(\boldsymbol{R})$ be a non-decreasing function such that

$$
\xi_{0}(t)=\left\{\begin{array}{l}
0 \text { for } t<-1 \\
1 \text { for } t>0
\end{array}\right.
$$

and we put in $r_{j} \leq r \leq r_{j+1}$

$$
\xi_{1}(r)=\xi_{0}\left(r-r_{j+1}\right)+j
$$

Then it is obvious that

$$
\left\{\begin{array}{l}
\lim _{r \rightarrow \infty} \xi_{1}(r)=\infty, \\
\xi_{1}(r) \leq \varphi_{0}(r)^{-1}, \\
0 \leq \xi_{1}^{\prime}(r) \leq \text { Const. }
\end{array}\right.
$$

We put $\xi_{2}(r)=\xi_{1}(\log r)$ and $\xi(r)=\sqrt{\xi_{2}(r)}$ for $r \geq e^{R_{0}}$ and $\xi(r) \equiv 1$ for $R_{0} \leq r$ $<e^{R_{0}}$. Then it is obvious that $\xi(r) \in C^{\infty}\left[R_{0}, \infty\right)$ and, (i) and (ii) hold. Since $\xi_{2}(r) \leq \xi_{1}(r) \leq \varphi_{0}(r)^{-1} \leq C_{4}^{-1} r \psi_{2}(r)^{-1} \sigma_{2}(r)$, we have $r^{-1} \psi_{2}(r) \sigma_{2}(r)^{-1} \xi(r) \leq$ $\sqrt{C_{4}^{-1} r^{-1} \psi_{2}(r) \sigma_{2}(r)^{-1}} \rightarrow 0$ as $r \rightarrow \infty$ by (E.4) so that we have (iii). By virtue of $\xi^{\prime}(r)=2^{-1} \xi_{2}(r)^{-1 / 2} \cdot \xi_{1}^{\prime}(\log r) \cdot r^{-1} \leq$ Const $r^{-1} \xi(r)^{-1} \leq$ Const $r^{-1}$, we have

$$
0 \leq r^{-1} \psi_{2}(r)^{2} \sigma_{2}(r)^{-1} \xi^{\prime}(r) \leq \text { Const } \sigma_{2}(r) \cdot\left\{r^{-1} \psi_{2}(r) \sigma_{2}(r)^{-1}\right\}^{2} \rightarrow 0
$$

as $r \rightarrow \infty$ by (E.1) and (E.4), which shows (iv).

Definition 5.3. Let $\xi(r)$ be the function given in Lemma 5.2. We put

$$
\phi_{0}(r)=\frac{\xi(r)}{r^{2}} \text { for } r \geq R_{0}
$$

Lemma 5.4. We have

$$
\lim _{r \rightarrow \infty} \psi_{2}^{2}(r) \sigma_{2}(r)^{-1}\left\{r^{2} \phi_{0}(r)^{2}+r\left|\phi_{0}\left(\gamma_{2}+(n-1) r^{-1}\right)+\phi_{0}^{\prime}(r)\right|\right\}=0 .
$$

Proof. Note $\gamma_{2}=\mathrm{O}\left(r^{-1}\right)$ by (E.1), (E.2) and Definition 3.6. By Lemma 5.2 we have

$$
\begin{aligned}
& \lim _{r \rightarrow \infty} r^{2} \psi_{2}^{2} \sigma_{2}^{-1} \phi_{0}^{2}=\lim _{r \rightarrow \infty}\left(r^{-1} \psi_{2} \sigma_{2}^{-1} \xi\right)^{2} \cdot \sigma_{2}=0, \\
& r \psi_{2}^{2} \sigma_{2}^{-1}\left|\phi_{0}\left(\gamma_{2}+(n-1) r^{-1}\right)+\phi_{0}^{\prime}\right| \\
& \quad \leq \text { Const } \psi_{2}^{2} \sigma_{2}^{-1}\left(r^{-2} \xi+r^{-1} \xi^{\prime}\right) \\
& \quad=\operatorname{Const}\left\{\left(r^{-1} \psi_{2} \sigma_{2}^{-1} \xi\right)^{2} \cdot \sigma_{2} \xi^{-1}+r^{-1} \psi_{2}^{2} \sigma_{2}^{-1} \xi^{\prime}\right\} \rightarrow 0 \text { as } r \rightarrow \infty
\end{aligned}
$$


so that we have the present lemma.

Lemma 5.5. There exists some constant $R_{4} \geq R_{3}$ such that for any $\rho(r)$ satisfying $\rho^{\prime}(r)>0$ in $\left[R_{3}, \infty\right)$ and for any $r \geq R_{4}$ we have

$$
\begin{aligned}
& G_{2}\left(x ; \rho, \phi_{0} ; v(\cdot ; \rho)\right) \\
& \geq\left(3 r \rho^{\prime}-C_{2} \sigma_{2}-1\right)\left|D_{r} v(x ; \rho)\right|^{2}+2^{-1} C_{3} \psi_{2}^{-2} \sigma_{2}|v(x ; \rho)|^{2} \\
& \quad+\left[r\left(\rho^{\prime 2}\right)^{\prime}+\eta_{2} \rho^{\prime 2}\right]|v(x ; \rho)|^{2}+r g_{2}\left\{\rho^{\prime \prime}+(n-1) r^{-1} \rho^{\prime}\right\}|v(x ; \rho)|^{2} \\
& \quad-r \rho^{\prime-1}\left|\rho^{\prime \prime}+\rho^{\prime} g_{2}+(n-1) r^{-1} \rho^{\prime}\right|^{2}|v(x ; \rho)|^{2},
\end{aligned}
$$

where $C_{2}$ and $C_{3}$ are positive constants given in Lemma 4.1.

Proof. We will write $v=v(x ; \rho)$ for short. By Definition 3.7 we have

$$
\begin{aligned}
G_{2}\left(x ; \rho, \phi_{0} ; v\right)= & G_{2}(x ; 0,0 ; v)+4 r \rho^{\prime}\left|D_{r} v\right|^{2} \\
& +2 r\left\{\rho^{\prime \prime}+\rho^{\prime} g_{2}+(n-1) r^{-1} \rho^{\prime}-\phi_{0}\right\} \operatorname{Re}\left[\bar{v} D_{r} v\right] \\
& +\left[\left\{r\left(\rho^{\prime 2}\right)^{\prime}+\eta_{2} \rho^{\prime 2}\right\}+r g_{2}\left\{\rho^{\prime \prime}+(n-1) r^{-1} \rho^{\prime}\right\}\right. \\
& -r\left\{\phi_{0}\left(\gamma_{2}+(n-1) r^{-1}\right)+\phi_{0}^{\prime}\right\} \cdot|v|^{2} \cdot
\end{aligned}
$$

By Lemma 4.1 (2) we have for any $r \geq R_{3}$

$$
G_{2}(x ; 0,0 ; v(\cdot ; \rho)) \geq-C_{2} \sigma_{2}\left|D_{r} v\right|^{2}+C_{3} \psi_{2}^{-2} \sigma_{2}|v|^{2} .
$$

By $\rho^{\prime}>0$ we have

$$
\begin{aligned}
& 2 r\left\{\rho^{\prime \prime}+\rho^{\prime} g_{2}+(n-1) r^{-1} \rho^{\prime}\right\} \operatorname{Re}\left[\bar{v} D_{r} v\right] \\
& \quad \geq-r \rho^{\prime}\left|D_{r} v\right|^{2}-r \rho^{\prime-1}\left|\rho^{\prime \prime}+\rho^{\prime} g_{2}+(n-1) r^{-1} \rho^{\prime}\right|^{2}|v|^{2}, \\
& -2 r \phi_{0} \operatorname{Re}\left[v D_{r} v\right] \geq-\left|D_{r} v\right|^{2}-r^{2} \phi_{0}^{2}|v|^{2} .
\end{aligned}
$$

These estimates and Lemma 5.4 give the assertion.

Definition 5.6. Let $\tau(r)$ be the functions given in Assumptions (E.6) and (E.7). We put

$$
\rho_{1}(r)=\int_{R_{0}}^{r}\left\{\exp \left(\int_{R_{0}}^{s} \frac{\tau(t)-\eta_{2}(t)}{2 t} d t\right)\right\} d s \text { for } r>R_{0}
$$

and

$$
M_{0}=\max \left\{C_{2}\left(\sup _{r \geq R_{3}} \sigma_{2}(r)\right)+1, \sup _{r \geq R_{3}} r\left|g_{2}(r)-g_{1}(r)\right|\right\}
$$

which is finite since $g_{i}(r)=\mathrm{O}\left(r^{-1}\right)$.

Lemma 5.7. We have

$$
\begin{aligned}
& \rho_{1}^{\prime}(r)>0 \text { for any } r>R_{0}, \\
& r \rho_{1}^{\prime}(r) \geq R \rho_{1}^{\prime}(R) \geq R_{0} \text { for any } r \geq R>R_{0},
\end{aligned}
$$




$$
m r \rho_{1}^{\prime}(r) \geq M_{0} \text { for any } r>R_{0} \text { and for any } m \geq R_{0}^{-1} M_{0},
$$

Proof. (5.5) is obvious. By (E.2) (or (E.2)') and (E.6) we have $\tau(r)>0$ and $\eta_{2}(r) \leq 2$ for any $r \geq R_{0}$. Then we have for any $r \geq R>R_{0}$

$$
\begin{aligned}
& r \rho_{1}^{\prime}(r)=R_{0} \exp \left(\int_{R_{0}}^{r}(2 t)^{-1}\left\{\tau(t)+2-\eta_{2}(t)\right\} d t\right) \\
& \quad \geq R_{0} \exp \left(\int_{R_{0}}^{R}(2 t)^{-1}\left\{\tau(t)+2-\eta_{2}(t)\right\} d t\right)=R \rho_{1}^{\prime}(R) \\
& \quad \geq R_{0} .
\end{aligned}
$$

Thus we have (5.6). (5.7) is obvious from (5.6).

Lemma 5.8. There exists some $R_{5}>R_{4}$ such that for any $r \geq R_{5}$ and any $m \geq R_{0}^{-1} M_{0}$ we have

$$
G_{2}\left(x ; m \rho_{1}, \phi_{0} ; v\left(\cdot ; m \rho_{1}\right)\right) \geq 0
$$

Proof. Let $m>0$. Since $\rho_{1}^{\prime}(r)>0$ for any $r \geq R_{3}$, we can use Lemma 5.5 with $\rho=m \rho_{1}$. Noting $\rho_{1}^{\prime \prime}(r)=(2 r)^{-1}\left\{\tau(r)-\eta_{2}(r)\right\} \rho_{1}^{\prime}(r)$, we have for any $r \geq$ $R_{4}$

$$
\begin{aligned}
G_{2}( & \left.x m \rho_{1}, \phi_{0} ; v\left(\cdot ; m \rho_{1}\right)\right) \\
\geq & \left(3 m r \rho_{1}^{\prime}-C_{2} \sigma_{2}-1\right)\left|D_{r} v\right|^{2} \\
\quad & +\left[2^{-1} C_{3} \psi_{2}^{-2} \sigma_{2}+m^{2} \tau \rho_{1}^{\prime 2}+m r g_{2} \rho_{1}^{\prime}\left\{(2 r)^{-1}\left(\tau-\eta_{2}\right)+(n-1) r^{-1}\right\}\right. \\
\quad & \left.-m r \rho_{1}^{\prime}\left|(2 r)^{-1}\left(\tau-\eta_{2}\right)+g_{2}+(n-1) r^{-1}\right|^{2}\right]|v|^{2} .
\end{aligned}
$$

By (5.4) and (5.7) we have for any $r>R_{3}$ and any $m \geq R_{0}^{-1} M_{0}$

$$
3 m r \rho_{1}^{\prime}-C_{2} \sigma_{2}-1 \geq 2 M_{0} \text {. }
$$

By (E.6), the boundedness of $\eta_{2}, \tau, r g_{2}$, there exist some constants $R_{6}>R_{4}$ and $C_{5}>0$ such that for any $r \geq R_{6}$ we have

$$
\begin{aligned}
& r \geq C_{1}^{-1} \sigma_{2}, \\
& r g_{2}\left\{(2 r)^{-1}\left(\tau-\eta_{2}\right)+(n-1) r^{-1}\right\} \geq-C_{5} r^{-1}, \\
& -r\left|(2 r)^{-1}\left(\tau-\eta_{2}\right)+g_{2}+(n-1) r^{-1}\right|^{2} \geq-C_{5} r^{-1} .
\end{aligned}
$$

Thus for any $r \geq R_{6}$ and any $m \geq R_{0}^{-1} M_{0}$ we have

$$
\begin{aligned}
& G_{2}\left(x ; m \rho_{1}, \phi_{0} ; v\left(\circ ; m \rho_{1}\right)\right) \\
& \quad \geq\left\{2^{-1} C_{3} \psi_{2}^{-2} \sigma_{2}+C_{1}^{-1} m^{2} \rho_{1}^{\prime 2} \sigma_{2}-2 m C_{5} r^{-1} \rho_{1}^{\prime}\right\}|v|^{2} \\
& \quad \geq\left\{2^{-1} C_{3} \psi_{2}^{-2} \sigma_{2}-C_{1} C_{5}^{2} r^{-2} \sigma_{2}^{-1}\right\}|v|^{2} \\
& \quad=\psi_{2}^{-2} \sigma_{2}\left\{2^{-1} C_{3}-C_{1} C_{5}^{2} r^{-2} \psi_{2}^{2} \sigma_{2}^{-2}\right\}|v|^{2} .
\end{aligned}
$$

By (E.4) there exists some $R_{5} \geq R_{6}$ such that for any $r \geq R_{5}$ and any $m \geq R_{0}^{-1} M_{0}$ we have (5.8). 
The following formula represents $J_{i}(x ; \rho, \phi ; v(\cdot ; \rho))$ in terms of $u$.

\section{Lemma 5.9.}

$$
\begin{aligned}
& J_{i}(x ; \rho, \phi ; v(\cdot ; \rho)) \\
& \quad=e^{2 \rho(r)}\left[2\left|D_{r} u\right|^{2}-|D u|^{2}+\left(2 \rho^{\prime}+g_{i}\right) \operatorname{Re}\left[\bar{u} D_{r} u\right]\right. \\
& \left.\quad+\left\{-q_{1}+2 \rho^{\prime 2}+g_{i} \rho^{\prime}+4^{-1} g_{i}^{2}-\phi\right\}|u|^{2}\right] .
\end{aligned}
$$

Proof. In Definition 3.7 (2), put $w=v(\cdot ; \rho)=e^{\rho} u$. By $D v=e^{\rho}\left\{\rho^{\prime} \hat{x} u\right.$ $+D u\}$, the above formula is easily shown.

Lemma 5.10. There exist $m_{0} \geq R_{0}^{-1} M_{0}$ and $R_{7} \geq R_{5}$ such that for any $t \geq$ $R_{7}$ we have

$$
F_{2}\left(t ; m_{0} \rho_{1}, \phi_{0} ; v\left(\cdot ; m_{0} \rho_{1}\right)\right)>0
$$

Proof. By Lemma 5.9 and Definition 3.7 (1),

$$
\exp \left\{-2 m \rho_{1}(t)\right\} F_{2}\left(t ; m \rho_{1}, \phi_{0} ; v\left(\cdot ; m \rho_{1}\right)\right)
$$

is a quadratic function of $m$ whose coefficient of $m^{2}$ is

$$
2 \int_{|x|=t} \Phi_{2}(r) \rho_{1}^{\prime 2}(r)|u|^{2} d S
$$

which is positive at some $t=R_{7} \geq R_{5}$ since the support of $u$ is not compact. Hence there exists some $m_{0} \geq R_{0}^{-1} M_{0}$ such that

$$
F_{2}\left(R_{7} ; m_{0} \rho_{1}, \phi_{0} ; v\left(\cdot ; m_{0} \rho_{1}\right)\right)>0 \text {. }
$$

Since $F_{2}$ is non-decreasing in $t \in\left[R_{5}, \infty\right)$ by Lemma 3.8 and Lemma 5.8, we have the assertion.

Our next aim is to define $\rho_{2}(r)$ such that (1.4) holds with $\rho=\rho_{2}$ and $\phi=$ $\phi_{0}$, that is to show Lemma 5.15.

Definition 5.11. Let $m_{0}$ be as in Lemma 5.10. For $R>R_{0}$, we put

$$
\rho_{2}(r)=\rho_{2}(r ; R)=C_{6}(R) \log r,
$$

where

$$
C_{6}(R)=m_{0} R \rho_{1}^{\prime}(R)
$$

Lemma 5.12. We have

$$
\rho_{2}^{\prime}(r)>0 \text { for any } r>R_{0},
$$




$$
\begin{aligned}
& \rho_{2}^{\prime}(R)=m_{0} \rho_{1}^{\prime}(R) \text { for any } R>R_{0}, \\
& M_{0} \leq C_{6}(R) \leq m_{0} r \rho_{1}^{\prime}(r) \text { for any } r \geq R>R_{0} .
\end{aligned}
$$

Proof. (5.10) is obvious from (5.5) and (5.9). (5.11) is obvious from (5.9). Noting $m_{0} \geq R_{0}^{-1} M_{0},(5.7)$ and (5.9) we have $C_{6}(R) \geq M_{0}$ for $R>R_{0}$. By (5.9) and (5.6) for any $r \geq R>R_{0}$ we have $C_{6}(R)=m_{0} R \rho_{1}^{\prime}(R) \leq m_{0} r \rho_{1}^{\prime}(r)$. Thus we have (5.12).

Lemma 5.13. There exists $R_{8} \geq R_{7}$ such that for any $r \geq R_{8}$ we have

$$
G_{2}\left(x ; \rho_{2}\left(\cdot ; R_{8}\right), \phi_{0} ; v\left(\cdot ; \rho_{2}\left(\cdot ; R_{8}\right)\right)\right) \geq 0 .
$$

Proof. By (5.10), Lemma 5.5 is applicable with $\rho=\rho_{2}(r ; R)$. Then for any $R \geq R_{7}$ and any $r \geq R$ we have

$$
\begin{aligned}
& G_{2}\left(x ; \rho_{2}(\cdot ; R), \phi_{0} ; v\left(\cdot ; \rho_{2}(\cdot ; R)\right)\right) \\
& \quad \geq\left(3 C_{6}(R)-C_{2} \sigma_{2}-1\right)\left|D_{r} v\right|^{2}+\left[2^{-1} C_{3} \psi_{2}^{-2} \sigma_{2}+C_{6}(R)^{2} r^{-2}\left(\eta_{2}-2\right)\right. \\
& \left.\quad+C_{6}(R)\left\{(n-2) r^{-1} g_{2}-\left|g_{2}+(n-2) r^{-1}\right|^{2}\right\}\right]\left|v\left(x ; \rho_{2}(\cdot ; R)\right)\right|^{2} .
\end{aligned}
$$

By the boundedness of $\eta_{2}$ and $r g_{2}$, there exists some $C_{7}>0$ such that for any $r$ $\geq R_{7}$ we have

$$
\begin{aligned}
& \eta_{2}-2 \geq-C_{7}, \\
& (n-2) r^{-1} g_{2}-\left|g_{2}+(n-2) r^{-1}\right|^{2} \geq-C_{7} r^{-2} .
\end{aligned}
$$

By (5.4) and (5.12) we have for any $R \geq R_{7}\left(>R_{3}\right)$ and any $r=|x| \geq R$

$$
3 C_{6}(R)-C_{2} \sigma_{2}(r)-1 \geq 2 M_{0} .
$$

Thus for any $R \geq R_{7}$ and $r \geq R$ we have

$$
\begin{aligned}
& G_{2}\left(x ; \rho_{2}(\cdot ; R), \phi_{0} ; v\left(\cdot ; \rho_{2}(\cdot ; R)\right)\right) \\
& \quad \geq\left\{2^{-1} C_{3} \psi_{2}^{-2} \sigma_{2}-C_{7} C_{6}(R)^{2} r^{-2}-C_{7} C_{6}(R) r^{-2}\right\}\left|v\left(x ; \rho_{2}(\cdot ; R)\right)\right|^{2} \\
& \quad \geq\left\{2^{-1} C_{3} \psi_{2}^{-2} \sigma_{2}-2 C_{7} C_{6}(R)^{2} r^{-2}-4^{-1} C_{7} r^{-2}\right\}\left|v\left(x ; \rho_{2}(\cdot ; R)\right)\right|^{2} .
\end{aligned}
$$

By (5.12), (5.3) and (E.7), there exists some $R_{8} \geq R_{7}$ such that for any $R \geq R_{8}$ and any $r \geq R$ we have

$$
\begin{aligned}
& 2 C_{7} C_{6}(R)^{2} r^{-2} \leq 2 m_{0}^{2} C_{7} \rho_{1}^{\prime}(r)^{2}=2 m_{0}^{2} C_{7} \exp \left(\int_{R_{0}}^{r} t^{-1}\left\{\tau(t)-\eta_{2}(t)\right\} d t\right) \\
& \leq 8^{-1} C_{3} \psi_{2}(r)^{-2} \sigma_{2}(r), \\
& 4^{-1} C_{7} r^{-2} \leq 8^{-1} C_{3} \psi_{2}(r)^{-2} \sigma_{2}(r) .
\end{aligned}
$$

Let $R=R_{8}$. Then for any $r \geq R_{8}$ we have

$$
G_{2}\left(x ; \rho_{2}\left(\cdot ; R_{8}\right), \phi_{0} ; v\left(\cdot ; \rho_{2}\left(\cdot, R_{8}\right)\right)\right) \geq 4^{-1} C_{3} \psi_{2}^{-2} \sigma_{2}\left|v\left(x ; \rho_{2}\left(\cdot ; R_{8}\right)\right)\right|^{2},
$$

which shows the present lemma. 
Definition 5.14. We put

$$
\rho_{2}(r)=\rho_{2}\left(r ; R_{8}\right)
$$

for short, where $R_{8}$ is the one given in Lemma 5.13.

Lemma 5.15. For any $t \geq R_{8}$ we have

$$
F_{2}\left(t ; \rho_{2}, \phi_{0} ; v\left(\cdot ; \rho_{2}\right)\right)>0 \text {. }
$$

Proof. Since $F_{2}\left(t ; \rho_{2}, \phi_{0} ; v\left(\cdot ; \rho_{2}\right)\right)$ is non-decreasing in $t \geq R_{8}$ by Lemmas 3.8 and 5.13 , it is sufficient to show $F_{2}\left(R_{8} ; \rho_{2}, \phi_{0} ; v\left(\cdot ; \rho_{2}\right)\right)>0$.

By Definition 3.7 and Lemma 5.9 we have

$$
\begin{aligned}
& F_{2}\left(r ; \rho_{2}, \phi_{0} ; v\left(\cdot ; \rho_{2}\right)\right) \\
& =\int_{|x|=r} \Phi_{2}(r) e^{2 \rho_{2}(r)}\left[2\left|D_{r} u\right|^{2}-|D u|^{2}+\left(2 \rho_{2}^{\prime}+g_{2}\right) \operatorname{Re}\left[\bar{u} D_{r} u\right]\right. \\
& \left.\quad+\left\{-q_{1}+2 \rho_{2}^{\prime 2}+q_{2} \rho_{2}^{\prime}+4^{-1} g_{2}^{2}-\phi_{0}\right\}|u|^{2}\right] d S \\
& =\exp \left[2\left\{\rho_{2}(r)-m_{0} \rho_{1}(r)\right\}\right] \\
& \quad \times \int_{|x|=r} \Phi_{2}(r) e^{2 m_{0} \rho_{1}(r)}\left[2\left|D_{r} u\right|^{2}-|D u|^{2}+\left(2 m_{0} \rho_{1}^{\prime}+g_{2}\right) \operatorname{Re}\left[\bar{u} D_{r} u\right]\right. \\
& \left.\quad+\left\{-q_{1}+2 m_{0}^{2} \rho_{1}^{\prime 2}+m_{0} g_{2} \rho_{1}^{\prime}+4^{-1} g_{2}^{2}-\phi_{0}\right\}|u|^{2}\right] d S \\
& \quad+\int_{|x|=r} \Phi_{2}(r) e^{2 \rho_{2}(r)}\left[2\left(\rho_{2}^{\prime}-m_{0} \rho_{1}^{\prime}\right) \operatorname{Re}\left[\bar{u} D_{r} u\right]\right. \\
& \left.\quad+\left\{2\left(\rho_{2}^{\prime 2}-m_{0}^{2} \rho_{1}^{\prime 2}\right)+\left(\rho_{2}^{\prime}-m_{0} \rho_{1}^{\prime}\right) g_{2}\right\}|u|^{2}\right] d S \\
& =\exp \left[2\left\{\rho_{2}(r)-m_{0} \rho_{1}(r)\right\}\right] \cdot F_{2}\left(r ; m_{0} \rho_{1}, \phi_{0} ; v\left(\cdot ; m_{0} \rho_{1}\right)\right) \\
& \quad+e^{2 \rho_{2}(r)}\left\{\rho_{2}^{\prime}(r)-m_{0} \rho_{1}^{\prime}(r)\right\} \int_{|x|=r} \Phi_{2}(r)\left[2 \operatorname{Re}\left[\bar{u} D_{r} u\right]\right. \\
& \left.\quad+\left\{2\left(\rho_{2}^{\prime}+m_{0} \rho_{1}^{\prime}\right)+g_{2}\right\}|u|^{2}\right] d S .
\end{aligned}
$$

Using $\rho_{2}^{\prime}\left(R_{8}\right)=m_{0} \rho_{1}^{\prime}\left(R_{8}\right)$ by (5.11) and using Lemma 5.10, we have

$$
\begin{aligned}
& F_{2}\left(R_{8} ; \rho_{2}, \phi_{0} ; v\left(\cdot ; \rho_{2}\right)\right) \\
& \quad=\exp \left[2\left\{\rho_{2}\left(R_{8}\right)-m_{0} \rho_{1}\left(R_{8}\right)\right\}\right] F_{2}\left(R_{8} ; m_{0} \rho_{1}, \phi_{0} ; v\left(\cdot ; m_{0} \rho_{1}\right)\right)>0,
\end{aligned}
$$

which is the desired result.

\section{§6. Proof of Theorem 1.1}

In this section we assume (B)-(E) and the validities of Lemmas 3.8 and 4.1. The following is obvious.

Lemma 6.1. One of the following two cases holds:

Case 1: There exists $R_{9} \geq R_{8}$ such that for any $r \geq R_{9}$ 


$$
\frac{d}{d r} \int_{|x|=r} r^{-1} \Phi_{1}(r)\left(2 \rho_{2}^{\prime}+g_{2}-g_{1}\right)|u|^{2} d S>0
$$

Case 2: For any $R_{10} \geq R_{8}$ there exists $R_{11} \geq R_{10}$ such that

$$
\frac{d}{d r} \int_{|x|=r} r^{-1} \Phi_{1}(r)\left(2 \rho_{2}^{\prime}+g_{2}-g_{1}\right)|u|^{2} d S \leq 0 \quad \text { at } r=R_{11}
$$

Lemma 6.2. In Case 1 of Lemma 6.1, Theorem 1.1 holds.

Proof. By virtue of (5.4) and (5.12), we have $2 \rho_{2}^{\prime}+g_{2}-g_{1} \geq M_{0} r^{-1}$ for any $r \geq R_{8}$. Since we are concerning with Case 1 and $u$ has not a compact support, there exists $R_{12} \geq R_{9}$ such that for any $r \geq R_{12}$ we have

$$
\begin{aligned}
0 & <\int_{|x|=R_{12}} r^{-1} \Phi_{1}(r)\left(2 \rho_{2}^{\prime}+g_{2}-g_{1}\right)|u|^{2} d S \\
& \leq \int_{|x|=r} r^{-1} \Phi_{1}(r)\left(2 \rho_{2}^{\prime}+g_{2}-g_{1}\right)|u|^{2} d S .
\end{aligned}
$$

On the other hand, since $\rho_{2}^{\prime}, g_{1}, g_{2}$ are of $\mathrm{O}\left(r^{-1}\right)$, we have

$$
\begin{aligned}
& \int_{|x|=r} r^{-1} \Phi_{1}(r)\left(2 \rho_{2}^{\prime}+g_{2}-g_{1}\right)|u|^{2} d S \\
\leq & \text { Const } \int_{|x|=r} \Phi_{1}(r) r^{-2}|u|^{2} d S \\
\leq & \text { Const } \int_{|x|=r} \Phi_{1}(r)\left[\left|D_{r} u\right|^{2}+\left\{r^{-2}+\left(q_{1}\right)_{-}\right\}|u|^{2}\right] d S,
\end{aligned}
$$

which yields the assertion.

In order to treat Case 2 given in Lemma 6.1, we prepare the next lemma.

\section{Lemma 6.3.}

$$
\begin{aligned}
& -\int_{|x|=r} r^{-1} \Phi_{1}\left(2 \rho_{2}^{\prime}+g_{2}-g_{1}\right) \operatorname{Re}\left[\bar{u} D_{r} u\right] d S \\
= & -\frac{1}{2} \frac{d}{d r} \int_{|x|=r} r^{-1} \Phi_{1}\left(2 \rho_{2}^{\prime}+g_{2}-g_{1}\right)|u|^{2} d S \\
& +\int_{|x|=r} r^{-1} \Phi_{1}\left[2^{-1}\left(2 \rho_{2}^{\prime}+g_{2}-g_{1}\right)\left\{\gamma_{1}+(n-2) r^{-1}\right\}\right. \\
& \left.+\rho_{2}^{\prime \prime}-r^{-1}\left(Q_{2}^{\prime}-Q_{1}^{\prime}\right)+r^{-2}\left(Q_{2}-Q_{1}\right)\right] \cdot|u|^{2} d S .
\end{aligned}
$$

Proof. Put $\phi=(2 r)^{-1} \Phi_{1}\left(2 \rho_{2}^{\prime}+g_{2}-g_{1}\right)$ and $w=u$ in (3.10). Noting $g_{2}-g_{1}$ $=-2 r^{-1}\left(Q_{2}-Q_{1}\right)$ by Definition 3.6, we have

$$
\frac{1}{2}\left(\int_{|x|=t}-\int_{|x|=s}\right)\left\{r^{-1} \Phi_{1}\left(2 \rho_{2}^{\prime}+g_{2}-g_{1}\right)|u|^{2}\right\} d S
$$




$$
\begin{aligned}
= & \int_{s<|x|<t}\left\{r^{-1} \Phi_{1}\left[\left(2 \rho_{2}^{\prime}+g_{2}-g_{1}\right) \operatorname{Re}\left[\bar{u} D_{r} u\right]\right\} d x\right. \\
& +\int_{s<|x|<t} r^{-1} \Phi_{1}\left[2^{-1}\left(2 \rho_{2}^{\prime}+g_{2}-g_{1}\right)\left\{\gamma_{1}+(n-2) r^{-1}\right\}\right. \\
& \left.+\rho_{2}^{\prime \prime}-r^{-1}\left(Q_{2}^{\prime}-Q_{1}^{\prime}\right)+r^{-2}\left(Q_{2}-Q_{1}\right)\right] \cdot|u|^{2} d x .
\end{aligned}
$$

Devide both sides by $t-s$, let $t \searrow s$ and put $s=r$. Then we have the assertion.

Lemma 6.4. In Case 2 of Lemma 6.1, Theorem 1.1 holds.

Proof. By (1) (2) of Definition 3.7, Lemmas 5.9 and 6.3 we have

$$
\begin{aligned}
& F_{1}(r ; 0,0 ; u) \\
= & \int_{|x|=r} \Phi_{1}(r)\left\{2\left|D_{r} u\right|^{2}-|D u|^{2}\right. \\
& \left.+g_{1}(r) \operatorname{Re}\left[\bar{u} D_{r} u\right]+\left(-q_{1}+4^{-1} g_{1}^{2}\right)|u|^{2}\right\} d S \\
= & \left(\frac{\Phi_{1}}{\Phi_{2}}\right)(r) e^{-2 \rho_{2}(r)} \int_{|x|=r} \Phi_{2}(r) e^{2 \rho_{2}(r)}\left[2\left|D_{r} u\right|^{2}-|D u|^{2}\right. \\
& +\left(2 \rho_{2}^{\prime}+g_{2}(r)\right) \operatorname{Re}\left[u D_{r} u\right] \\
& \left.+\left\{-q_{1}+2 \rho_{2}^{\prime 2}+g_{2} \rho_{2}^{\prime}+4^{-1} g_{2}^{2}-\phi_{0}\right\}|u|^{2}\right] d S \\
& -\int_{|x|=r} \Phi_{1}(r)\left(2 \rho_{2}^{\prime}+g_{2}-g_{1}\right) \operatorname{Re}\left[u D_{r} u\right] d S \\
& +\int_{|x|=r} \Phi_{1}(r)\left[\phi_{0}-\left\{4^{-1}\left(g_{2}^{2}-g_{1}^{2}\right)+2 \rho_{2}^{\prime 2}+g_{2} \rho_{2}^{\prime}\right\}\right]|u|^{2} d S \\
= & \left(\frac{\Phi_{1}}{\Phi_{2}}\right)(r) e^{-2 \rho_{2}(r)} F_{2}\left(r ; \rho_{2}, \phi_{0} ; v\left(\cdot ; \rho_{2}\right)\right) \\
& -\frac{r}{2} \frac{d}{d r} \int_{|x|=r} r^{-1} \Phi_{1}\left(2 \rho_{2}^{\prime}+g_{2}-g_{1}\right)|u|^{2} d S \\
& +\int_{|x|=r} \Phi_{1}(r)\left[\phi_{0}-\left\{4^{-1}\left(g_{2}^{2}-g_{1}^{2}\right)+2 \rho_{2}^{\prime 2}+g_{2} \rho_{2}^{\prime}\right\}\right. \\
& +2^{-1}\left(2 \rho_{2}^{\prime}+g_{2}-g_{1}\right)\left\{\gamma_{1}+(n-2) r^{-1}\right\} \\
& \left.+\rho_{2}^{\prime \prime}-r^{-1}\left(Q_{2}^{\prime}-Q_{1}^{\prime}\right)+r^{-2}\left(Q_{2}-Q_{1}\right)\right]|u|^{2} d S .
\end{aligned}
$$

By $g_{i}=\mathrm{O}\left(r^{-1}\right)$ and $\rho_{2}^{\prime}=\mathrm{O}\left(r^{-1}\right)$, we have

$$
4^{-1}\left(g_{2}^{2}-g_{1}^{2}\right)+2 \rho_{2}^{\prime 2}+g_{2} \rho_{2}^{\prime}=\mathrm{O}\left(r^{-2}\right) .
$$

By $\gamma_{1}=\mathrm{O}\left(r^{-1}\right)$, we have

$$
2^{-1}\left(2 \rho_{2}^{\prime}+g_{2}-g_{1}\right)\left\{\gamma_{1}+(n-2) r^{-1}\right\}=\mathrm{O}\left(r^{-2}\right) .
$$

By $Q_{i}=\mathrm{O}(1), \rho_{2}^{\prime \prime}=\mathrm{O}\left(r^{-2}\right)$ and (E.5), we have

$$
\rho_{2}^{\prime \prime}-r^{-1}\left(Q_{2}^{\prime}-Q_{1}^{\prime}\right)+r^{-2}\left(Q_{2}-Q_{1}\right)=\mathrm{O}\left(r^{-2}\right) .
$$


Thus, using $\phi_{0}(r)=\xi(r) r^{-2}$ by Definition 5.3 and $\lim _{r \rightarrow \infty} \xi(r)=\infty$ by Lemma 5.2 (ii), there exists some $R_{13} \geq R_{0}$ such that for any $r \geq R_{13}$ we have

$$
\begin{aligned}
\phi_{0}- & 4^{-1}\left(g_{2}^{2}-g_{1}^{2}\right)+2 \rho_{2}^{\prime 2}+g_{2} \rho_{2}^{\prime 2} \\
& +2^{-1}\left(2 \rho_{2}^{\prime}+g_{2}-g_{1}\right)\left\{\gamma_{1}+(n-2) r^{-1}\right\} \\
& +\rho_{2}^{\prime \prime}-r^{-1}\left(Q_{2}^{\prime}-Q_{1}^{\prime}\right)+r^{-2}\left(Q_{2}-Q_{1}\right) \\
> & 0 .
\end{aligned}
$$

In the statement of Case 2 of Lemma 6.1 let $R_{10}=\max \left\{R_{8}, R_{13}\right\}$. Since Case 2 holds, we have by (6.3) and (6.4) for some $R_{11} \geq R_{10}$

$$
F_{1}\left(R_{11} ; 0,0 ; u\right) \geq\left[\left(\frac{\Phi_{1}}{\Phi_{2}}\right)(r) e^{-2 \rho_{2}(r)} F_{2}\left(r ; \rho_{2}, \phi_{0} ; v\left(\cdot ; \rho_{2}\right)\right]_{r=R_{11}},\right.
$$

the right hand side of which is positive by Lemma 5.15. This proves Theorem 1.1 by Proposition 5.1 with $R_{*}=R_{11}$.

\section{§7. Proof of Theorem 1.2}

Lemma 7.1. Assume that $\sigma_{1}(r)$ and $\eta_{1}(r)$ are bounded functions and $p(r) \in C^{0}\left[R_{0}, \infty\right)$ is a non-decreasing positive function satisfying (G.2). Then there exist some constants $R_{14} \geq R_{0}$ and $C_{8}>0$ such that for any $R \geq R_{14}$ we have

$$
\int_{R+(1 / 3)}^{R+(2 / 3)} p(r)^{-1} \Phi_{1}(r)^{-1} d r \geq C_{8} p(R)^{-1} \Phi_{1}(R)^{-1}
$$

Proof. By the boundedness of $\sigma_{1}(r)$ and $\eta_{1}(r)$ and by Definition 3.6 there exists some constant $C_{9}>0$ such that for any $t \geq R_{0}$ we have $\left|\gamma_{1}(t)\right| \leq C_{9} t^{-1}$. Then for any $R \geq R_{0}$ and any $R \leq r \leq R+1$ we have

$$
\begin{aligned}
\Phi_{1}(r) & =\Phi_{1}(R) \exp \left(\int_{R}^{r} \gamma_{1}(t) d t\right) \leq \Phi_{1}(R) \exp \left(\int_{R}^{R+1}\left|\gamma_{1}(t)\right| d t\right) \\
& \leq \Phi_{1}(R)(R+1)^{C_{9}} R^{-C_{9}} .
\end{aligned}
$$

Noting that $p(r)$ is non-decreasing in $\left[R_{0}, \infty\right)$, we have for any $R \geq R_{0}$ and any $R \leq r \leq R+1$

$$
p(r)^{-1} \Phi_{1}(r)^{-1} \geq p(R)^{-1} \Phi_{1}(R)^{-1} \cdot p(R) p(R+1)^{-1} \cdot R^{C_{9}}(R+1)^{-C_{9}} .
$$

By $\liminf _{R \rightarrow \infty} p(R) p(R+1)^{-1}>0$ and $\lim _{R \rightarrow \infty} R(R+1)^{-1}=1$, there exists some constants $C_{8}>0$ and $R_{14} \geq R_{0}$ such that for any $R \geq R_{14}$ and any $R \leq r \leq R+1$ we have

$$
p(r)^{-1} \Phi_{1}(r)^{-1} \geq 3 C_{8} p(R)^{-1} \Phi_{1}(R)^{-1},
$$


from which we can easily have the assertion.

Lemma 7.2. Assume (B)-(D) and assume that there exist some constant 0 $<d_{1}<1$, some positive function $\tilde{D}(x) \in C^{0}(\bar{\Omega})$ and some real-valued bounded function $Q_{0}(x) \in C^{1}(\bar{\Omega})$ such that

(i) for any $w(x) \in C_{0}^{\infty}(\Omega)$ we have

$$
\begin{gathered}
\int_{\Omega}\left\{\left(q_{1}\right)_{-}(x)+\left(\operatorname{Re}\left[q_{2}\right]-r^{-1} \partial_{r} Q_{0}\right)_{-}(x)\right\}|w(x)|^{2} d x \\
\leq \int_{\Omega}\left\{d_{1}|\nabla w(x)|^{2}+\tilde{D}(x)|w(x)|^{2}\right\} d x
\end{gathered}
$$

(ii) $\tilde{p}(x) \geq$ Const $r^{-2}$ for any $r \geq R_{0}$.

Then there exist some constants $R_{15} \geq R_{0}$ and $C_{10}>0$ such that for any real-valued function $\phi(r) \in C_{0}^{1}\left(R_{15}, \infty\right)$ we have

$$
\begin{aligned}
& \int_{\Omega} \psi(r)^{2}\left[|D u|^{2}+\left\{\left(q_{1}\right)_{-}(x)+\left(\operatorname{Re}\left[q_{2}\right]-r^{-1} \partial_{r} Q_{0}\right)_{-}(x)\right\}|u|^{2}\right] d x \\
& \quad \leq C_{10} \int_{\Omega}\left\{\tilde{p}(x) \psi(r)^{2}+\psi^{\prime}(r)^{2}\right\}|u|^{2} d x .
\end{aligned}
$$

Proof. By the boundedness of $Q_{0}(x) \in C^{1}(\bar{\Omega})$ and $\sigma_{i}(r)$, and by $0<d_{1}<$ 1 , there exist some $R_{15} \geq R_{0}, 0<\varepsilon<1$ and $C_{11}>0$ such that for any $r \geq R_{15}$ we have

$$
\begin{aligned}
& 1-\varepsilon-(1+\varepsilon) d_{1}>0, \\
& \left(2 \varepsilon^{-1}+1\right)\left(Q_{0}^{2}+1\right)+|n-2|\left|Q_{0}\right| \leq C_{11} .
\end{aligned}
$$

Noting $\operatorname{Re}\left[\bar{u} \partial_{r} u\right]=\operatorname{Re}\left[\bar{u} D_{r} u\right]$ by (B), we have by integration by parts

$$
\begin{aligned}
& \int_{\Omega} r^{-1} \psi(r)^{2} \partial_{r} Q_{0} \cdot|u|^{2} d x \\
& =-\int_{\Omega}\left[2 r^{-1} \psi^{2} Q_{0} \operatorname{Re}\left[\bar{u} \partial_{r} u\right]+\left\{2 r^{-1} \psi \psi^{\prime}+(n-2) r^{-2} \psi^{2}\right\} Q_{0}|u|^{2}\right] d x \\
& =-\int_{\Omega}\left[2 r^{-1} \phi^{2} Q_{0} \operatorname{Re}\left[\bar{u} D_{r} u\right]+\left\{2 r^{-1} \psi \psi^{\prime}+(n-2) r^{-2} \psi^{2}\right\} Q_{0}|u|^{2}\right] d x
\end{aligned}
$$

On the other hand, since $u(x)$ satisfies $(0.1)$ and $\psi(r) \in C_{0}^{1}\left(R_{15}, \infty\right)$, we have by integration by parts

$$
\begin{aligned}
0 & =\operatorname{Re} \int_{\Omega}\{-\langle D, D u\rangle+q u\} \phi^{2} \bar{u} d x \\
& =\int_{\Omega}\left\{\psi^{2}|D u|^{2}+2 \psi \psi^{\prime} \operatorname{Re}\left[\bar{u} D_{r} u\right]+\psi^{2} \operatorname{Re}[q]|u|^{2}\right\} d x
\end{aligned}
$$

From these two relations we have

$$
0=\int_{\Omega}\left[\psi^{2}|D u|^{2}+2 \psi\left\{\psi^{\prime}-r^{-1} \psi Q_{0}\right\} \operatorname{Re}\left[\bar{u} D_{r} u\right]\right.
$$




$$
\left.+\left\{\psi^{2}\left(\operatorname{Re}[q]-r^{-1} \partial_{r} Q_{0}\right)-2 r^{-1} \psi \psi^{\prime} Q_{0}-(n-2) r^{-2} \psi^{2} Q_{0}\right\}|u|^{2}\right] d x
$$

Noting the above relation and using the following inequalities

$$
\begin{aligned}
& 2 \psi\left\{\psi^{\prime}-r^{-1} \psi Q_{0}\right\} \operatorname{Re}\left[\bar{u} D_{r} u\right] \\
& \quad \geq-\varepsilon \psi^{2}\left|D_{r} u\right|^{2}-\varepsilon^{-1}\left|\psi^{\prime}-r^{-1} \psi Q_{0}\right|^{2}|u|^{2} \\
& \quad \geq-\varepsilon \psi^{2}|D u|^{2}-2 \varepsilon^{-1}\left(\psi^{\prime 2}+r^{-2} \psi^{2} Q_{0}^{2}\right)|u|^{2} \\
& \operatorname{Re}[q]-r^{-1} \partial_{r} Q_{0} \geq-\left\{\left(q_{1}\right)_{-}+\left(\operatorname{Re}\left[q_{2}\right]-r^{-1} \partial_{r} Q_{0}\right)_{-}\right\} \\
& -2 r^{-1} \psi \psi^{\prime} Q_{0} \geq-\left\{\psi^{\prime 2}+r^{-2} \psi^{2} Q_{0}^{2}\right\}
\end{aligned}
$$

we have

$$
\begin{aligned}
& (1-\varepsilon) \int_{\Omega} \psi^{2}|D u|^{2} d x \\
& \leq \int_{\Omega} \psi^{2}\left\{\left(q_{1}\right)_{-}+\left(\operatorname{Re}\left[q_{2}\right]-r^{-1} \partial_{r} Q_{0}\right)_{-}\right\}|u|^{2} d x \\
& \quad+C_{11} \int_{\Omega}\left\{\psi^{\prime 2}+r^{-2} \psi^{2}\right\}|u|^{2} d x .
\end{aligned}
$$

By (C.2), (D.2), $Q_{0} \in C^{1}(\bar{\Omega})$ and the limiting procedure, we have (7.1) for any $w \in H^{1}(\Omega)$ with a compact support.

Now for any $\eta>0$ let $u_{\eta}(x)=\left\{|u(x)|^{2}+\eta^{2}\right\}^{1 / 2}$. Since $|u| \leq\left|u_{\eta}\right|$ and $u_{\eta} \nabla u_{\eta}$ $=\operatorname{Re}[\bar{u} D u]=\operatorname{Re}[\bar{u} D u]$, we have

$\left|\nabla u_{\eta}\right| \leq|D u|$,

$\psi(r) u_{\eta}(x) \in H^{1}(\Omega)$,

$\operatorname{supp}\left[\phi(r) u_{\eta}(x)\right]$ is a compact set in $\Omega$.

Hence we have

$$
\begin{aligned}
& \int_{\Omega}\left\{\left(q_{1}\right)_{-}+\left(\operatorname{Re}\left[q_{2}\right]-r^{-1} \partial_{r} Q_{0}\right)_{-}\right\} \psi^{2}\left|u_{\eta}\right|^{2} d x \\
& \leq d_{1} \int_{\Omega}\left|\nabla\left(\phi u_{\eta}\right)\right|^{2} d x+\int_{\Omega} \tilde{p} \psi^{2}\left|u_{\eta}\right|^{2} d x \\
& \leq(1+\varepsilon) d_{1} \int_{\Omega} \psi^{2}|D u|^{2} d x+\int_{\Omega}\left\{\left(1+\varepsilon^{-1}\right) d_{1} \psi^{2}+\tilde{p} \psi^{2}\right\}\left(|u|^{2}+\eta^{2}\right) d x .
\end{aligned}
$$

Letting $\eta \downarrow 0$, we have

$$
\begin{aligned}
& \int_{\Omega} \psi^{2}\left\{\left(q_{1}\right)_{-}+\left(\operatorname{Re}\left[q_{2}\right]-r^{-1} \partial_{r} Q_{0}\right)_{-}\right\}|u|^{2} d x \\
& \quad \leq(1+\varepsilon) d_{1} \int_{\Omega} \psi^{2}|D u|^{2} d x+\int_{\Omega}\left\{\left(1+\varepsilon^{-1}\right) d_{1} \psi^{\prime 2}+\tilde{D} \psi^{2}\right\}|u|^{2} d x
\end{aligned}
$$

Then by (7.2) and (7.3) we have

$$
(1-\varepsilon) \int_{\Omega} \psi^{2}|D u|^{2} d x
$$




$$
\begin{aligned}
\leq & (1+\varepsilon) d_{1} \int_{\Omega} \psi^{2}|D u|^{2} d x \\
& +\int_{\Omega}\left[\left\{\left(1+\varepsilon^{-1}\right) d_{1}+C_{11}\right\}{\psi^{\prime}}^{2}+\left\{C_{11} r^{-2}+\tilde{p}\right\} \psi^{2}\right]|u|^{2} d x .
\end{aligned}
$$

Noting $\bar{D}(x) \geq$ Const $r^{-2}$ and $1-\varepsilon>(1+\varepsilon) d_{1}$, there exists some constant $C_{12}>$ 0 such that for any $\psi \in C_{0}^{\infty}\left(R_{15}, \infty\right)$ we have

$$
\int_{\Omega} \psi^{2}|D u|^{2} d x \leq C_{12} \int_{\Omega}\left\{\psi^{2}+\tilde{D} \psi^{2}\right\}|u|^{2} d x .
$$

Using (7.3) again, we have the assertion.

Proof of Theorem 1.2. By Theorem 1.1 there exist some constants $R_{16} \geq$ $\max \left\{R_{14}, R_{15}\right\}$ and $C_{13}>0$ such that for any $r \geq R_{16}$ we have

$$
C_{13} \Phi_{1}(r)^{-1} \leq \int_{|x|=r}\left[\left|D_{r} u\right|^{2}+\left\{r^{-2}+\left(q_{1}\right)_{-}\right\}|u|^{2}\right] d S .
$$

Let $R \geq R_{16}$ and $\psi_{R}(r) \in C_{0}^{\infty}(R, R+1)$ be such that $\psi_{R}(r)=1$ in $R+(1 / 3)$ $\leq r \leq R+(2 / 3), 0 \leq \psi_{R}(r) \leq 1$ in $R<r<R+1$ and $\left|\psi_{R}^{\prime}(r)\right| \leq$ Const.

Multiply both sides of (7.4) by $p(r)^{-1}$ and integrate them in $R+(1 / 3) \leq r$ $\leq R+(2 / 3)$. Then, noting (G.2), Lemma 7.1 gives

$$
\begin{aligned}
& C_{8} C_{13} p(R)^{-1} \Phi_{1}(R)^{-1} \\
& \quad \leq \int_{R+(1 / 3)<|x|<R+(2 / 3)} p(r)^{-1}\left[|D u|^{2}+\left\{r^{-2}+\left(q_{1}\right)_{-}\right\}|u|^{2}\right] d x \\
& \quad \leq \int_{\Omega} \psi_{R}(r)^{2} p(r)^{-1}\left[|D u|^{2}+\left\{r^{-2}+\left(q_{1}\right)_{-}\right\}|u|^{2}\right] d x .
\end{aligned}
$$

Since we have $p(r) \geq p\left(R_{0}\right) \geq$ Const $r^{-2}$ for any $r \geq R_{0}$ by the non-decrease of $p(r), p(r)$ satisfies the assumption of Lemma 7.2 with $\bar{p}=p$ by (G.1). Applying Lemma 7.2 with $\psi(r)=\phi_{R}(r) p(r)^{-1 / 2}$, and noting that $p$ is non-decreasing and by (G.3) $p^{\prime 2} p^{-3}$ is bounded on $\left[R_{0}, \infty\right)$, there exist some $R_{17} \geq R_{16}$ and $C_{14}>0$ such that for any $R \geq R_{17}$ we have

$$
\begin{aligned}
C_{8} & C_{13} p(R)^{-1} \Phi_{1}(R)^{-1} \\
\leq & C_{10} \int_{R<|x|<R+1}\left[\psi_{R}(r)^{2}+2 p(r)^{-1}\left\{\psi_{R}^{\prime}(r)^{2}+4^{-1} \psi_{R}(r)^{2} p^{\prime}(r)^{2} p(r)^{-2}\right\}\right]|u|^{2} d x \\
& +\int_{R<|x|<R+1} \psi_{R}(r)^{2} p(r)^{-1} r^{-2}|u|^{2} d x \\
\text { (7.5) } \leq & C_{14} \int_{R<|x|<R+1}|u|^{2} d x,
\end{aligned}
$$

which yields the assertion of Theorem 1.2 (1).

Integrating of (7.5) gives 


$$
\begin{aligned}
& C_{8} C_{13} \int_{R_{17}}^{\infty} p(R)^{-1} \Phi_{1}(R)^{-1} d R \\
& \quad \leq C_{14} \int_{R_{17}}^{\infty} d R \int_{R<|x|<R+1}|u|^{2} d x \\
& \quad \leq C_{14} \int_{|x|>R_{17}}|u|^{2} d x,
\end{aligned}
$$

which yields the assertion of Theorem 1.2 (2).

\section{§8. Generalization to the Second Order Elliptic Problem}

In this section we consider a solution $u(x) \in H_{l o c}^{2}(\Omega)$ of a second order elliptic equation

$$
\begin{gathered}
-\sum_{j, k=1}^{n}\left(\partial_{j}+\sqrt{-1} b_{j}(x)\right) a_{j k}(x)\left(\partial_{k}+\sqrt{-1} b_{k}(x)\right) u(x) \\
+\left(q_{1}(x)+q_{2}(x)\right) u(x)=0
\end{gathered}
$$

for $x \in \Omega:=\left\{x\left|x \in \mathbb{R}^{n},\right| x \mid>R_{0}\right\}$.

The aim of this section is to extend the results of $\S 1$ in two directions :

(i) to introduce the coefficients $a_{j k}(x)$;

(ii) to admit for $\psi_{i}, \sigma_{i}$ and $\eta_{i}$ to depend on $x$, which are assumed to depend on $r$ only in $\S 1$.

In a similar way which has been applied to the nontrivial solution $u$ of (0.1) in $\$ 3-\$ 7$, we can obtain the following results to the solution $u$ of (8.1). We omit the proofs.

Assumptions. Let us consider the equation (8.1). We assume that there exist some real-valued functions $\phi_{i}(x), \sigma_{i}(x), \eta_{i}(x) \in C^{0}(\bar{\Omega})(i=1,2), \tau(r) \in$ $C^{0}\left[R_{0}, \infty\right)$ and some constants $a_{i}, b_{i}, c_{i}(i=1,2)$ satisfying the following conditions (A.1)-(F.4) :

(A.1) $A(x)=\left(a_{j k}(x)\right)$ is an $n \times n$ real symmetric matrix whose element is of class $C^{2}(\bar{\Omega})$;

(A.2) there exists some constant $C_{15} \geq 1$ such that for any $x \in \bar{\Omega}$ and any $\xi \in$ $C^{n}$

$$
C_{15}^{-1}|\xi|^{2} \leq\langle\xi, A(x) \bar{\xi}\rangle \leq C_{15}|\xi|^{2} ;
$$

(A.3) $\lim _{|x| \rightarrow \infty} \sigma_{i}(x)^{-1}\left(a_{j k}(x)-\delta_{j k}\right)=0$, where $\delta_{j k}$ is the Kronecker's delta ;

(A.4) $\lim _{|x| \rightarrow \infty} r \sigma_{i}(x)^{-1} \partial_{j} a_{k l}(x)=0$;

(A.5) $\lim _{|x| \rightarrow \infty} r \psi_{i}(x) \sigma_{i}(x)^{-1} \partial_{j} \partial_{k} a_{l m}(x)=0$; 
(B.1) each $b_{j}(x)$ is a real-valued function;

(B.2) for any $w(x) \in H_{l o c}^{1}(\Omega)$ we have $b_{j}(x) w(x),\left(\partial_{j} b_{k}(x)\right) w(x) \in L_{l o c}^{2}(\Omega)$;

(C.1) $\quad q_{1}(x)$ is a real-valued function;

(C.2) for any $w(x) \in H_{\text {loc }}^{1}(\Omega)$ we have $q_{1}(x)|w(x)|^{2} \in L_{l o c}^{1}(\Omega)$;

(C.3) for any $w(x) \in H_{l o c}^{1}(\Omega)$ we have $\left\langle\hat{x}, A D q_{1}(x)\right\rangle|w(x)|^{2} \in L_{l o c}^{1}(\Omega)$;

(D.1) $q_{2}(x)$ may be a complex-valued function;

(D.2) for any $w(x) \in H_{l o c}^{1}(\Omega)$ we have $q_{2}(x)|w(x)|^{2} \in L_{l o c}^{1}(\Omega)$;

(E.1) $\sigma_{i}(x)$ is bounded in $\bar{\Omega}$ and $\sigma_{i}(x)>0(i=1,2)$ for any $r \geq R_{0}$;

(E.2) $\eta_{i}(x)$ is bounded in $\bar{\Omega}$ and we have

$$
\sup _{|x| \geq R_{0}} \eta_{i}(x)<2(i=1,2) \text {; }
$$

(E.3) $\quad \phi_{i}(x)>0(i=1,2)$ for any $r \geq R_{0}$;

(E.4) $\lim _{|x| \rightarrow \infty} r^{-1} \psi_{i}(x) \sigma_{i}(x)^{-1}=0(i=1,2)$;

(E.5) $\sigma_{1}(x)+\eta_{1}(x)-\left(\sigma_{2}(x)+\eta_{2}(x)\right)$ depends only on $r=|x|$;

(E.6) (i) $\sigma_{i}(x)-\eta_{i}(x) \in C^{1}(\bar{\Omega})(i=1,2)$,

(ii) $r\left\langle\bar{x}, A D\left\{\left(\sigma_{1}(x)-\eta_{1}(x)\right)-\left(\sigma_{2}(x)-\eta_{2}(x)\right)\right\}\right\rangle$ is bounded in $\bar{\Omega}$;

(E.7) there exists some constant $C_{16} \geq 1$ such that for any $r \geq R_{0}$

$$
C_{16}^{-1} \sigma_{2}(x) \leq \tau(|x|) \leq C_{16}
$$

(E.8) $\lim _{|x| \rightarrow \infty} \psi_{2}(x)^{2} \sigma_{2}(x)^{-1} \exp \left(\int_{R_{0}}^{|x|} \frac{\tau(t)-\eta_{*}(t)}{t} d t\right)=0$,

where we put

$$
\eta_{*}(r)=\inf _{|x|=r} \eta_{2}(x)
$$

(F.1) $\quad a_{1}>1, a_{2}>0, b_{i}>1(i=1,2)$;

(F.2) $\quad \limsup _{|x| \rightarrow \infty} \phi_{i}(x)^{2} \sigma_{i}(x)^{-1}\left[r\langle\bar{x}, A \hat{x}\rangle^{-1}\left\langle\hat{x}, A D q_{1}\right\rangle+\eta_{i}(x) q_{1}\right.$

$$
\begin{aligned}
& +a_{i} \sigma_{i}(x)^{-1}\langle\bar{x}, A \bar{x}\rangle^{-1}\left|r q_{2}-\left\langle\bar{x}, A D Q_{i}(x)\right\rangle\right|^{2} \\
& +b_{i}\left(2-\eta_{i}\right)^{-1}\left\{\left\langle D Q_{i}, A D Q_{i}\right\rangle-\langle\bar{x}, A \bar{x}\rangle^{-1}\left|\left\langle\bar{x}, A D Q_{i}\right\rangle\right|^{2}\right. \\
& \left.\left.+\langle\bar{x}, A \bar{x}\rangle^{-2} r^{2}\langle B A \bar{x}, A B A \bar{x}\rangle\right\}\right]<0(i=1,2),
\end{aligned}
$$

where we put 

(i) $\int_{R_{0}}^{|x|} \frac{\sigma_{i}(t \hat{x})+\eta_{i}(t \hat{x})}{2 t} d t \in C_{1}(\bar{\Omega})$
(ii) there exist some constants $c_{i}(i=1,2)$ such that for any $x \in \bar{\Omega}$ we have

$$
\begin{gathered}
0<c_{1}<\left(1-a_{1}^{-1}\right)\left(1-b_{1}^{-1}\right), c_{2}>0 \text { and } \\
\left\langle\left(D-\hat{x} \partial_{r}\right) \int_{R_{0}}^{|x|} \frac{\sigma_{i}(t \hat{x})+\eta_{i}(t \hat{x})}{2 t} d t, A\left(D-\hat{x} \partial_{r}\right) \int_{R_{0}}^{|x|} \frac{\sigma_{i}(t \hat{x})+\eta_{i}(t \hat{x})}{2 t} d t\right\rangle \\
\leq c_{i} r^{-2} \sigma_{i}(x)\left\{2-\eta_{i}(x)\right\}\langle\hat{x}, A \hat{x}\rangle(i=1,2)
\end{gathered}
$$

$$
\text { (i) }\left\langle\left(\nabla-\widehat{x} \partial_{r}\right) \int_{R_{0}}^{r} \frac{\sigma_{i}(t \hat{x})+\eta_{i}(t \hat{x})}{t} d t, A \bar{x}\right\rangle \in C^{1}(\bar{\Omega}) \text {, }
$$

(ii) $\lim _{|x| \rightarrow \infty} r \psi_{i}(x) \sigma_{i}(x)^{-1} \nabla\left(\left\langle\left(\nabla-\hat{x} \partial_{r}\right) \int_{R_{0}}^{r} \frac{\sigma_{i}(t \hat{x})+\eta_{i}(t \hat{x})}{t} d t, A \hat{x}\right\rangle\right)=0$

$$
(i=1,2) \text {. }
$$

As compared with the Assumptions in $\$ 1$, we add the Assumption (A), do not change (B)-(D) essentially and do change (E)-(F) slightly.

Our main results are as follows :

Theorem 8.1. Let $u(x) \in H_{\text {loc }}^{2}(\Omega)$ satisfy the equation (8.1). Assume that (A)-(F) hold. If $\operatorname{supp}[u]$ is not a compact set in $\bar{\Omega}$, then we have

$$
\liminf _{R \rightarrow \infty} \int_{|x|=R} \Phi_{1}(x)\left[|\langle\hat{x}, A D u\rangle|^{2}+\left\{r^{-2}+\left(q_{1}\right)_{-}\right\}|u|^{2}\right] d S>0,
$$

where

$$
\Phi_{1}(x)=\exp \left(\int_{R_{0}}^{|x|} \frac{\sigma_{1}(t \hat{x})+\eta_{1}(t \hat{x})}{2 t} d t\right)
$$

Theorem 8.2. In addition to the assumptions of Theorem 8.1, assume that

(G) there exist some real-valued bounded function $Q_{0}(x) \in C^{1}(\bar{\Omega})$, some positive and non-decreasing function $p(r) \in C^{1}\left[R_{0}, \infty\right)$ and some constant $0<$ $d_{1}<1$ satisfying the following (G.1)-(G.3):

(G.1) for any $w(x) \in C_{0}^{\infty}(\Omega)$ we have

$$
\begin{aligned}
& \int_{\Omega}\left\{\left(q_{1}\right)_{-}(x)+\left(\operatorname{Re}\left[q_{2}\right]-r^{-1}\left\langle\bar{x}, A D Q_{0}\right\rangle\right)_{-}(x)\right\}|w(x)|^{2} d x \\
& \quad \leq \int_{\Omega}\left\{d_{1}|\nabla w(x)|^{2}+p(r)|w(x)|^{2}\right\} d x
\end{aligned}
$$


(G.2) $\liminf _{r \rightarrow \infty} p(r) p(r+1)^{-1}>0$;

(G.3) $p^{\prime}(r)^{2} p(r)^{-3}$ is bounded in $\left[R_{0}, \infty\right)$.

Then we have

(1) $\quad \liminf _{R \rightarrow \infty} p(R) \Phi^{*}(R) \int_{R<|x|<R+1}|u(x)|^{2} d x>0$;

(2) moreover if $\int_{R_{0}}^{\infty} p(R)^{-1} \Phi^{*}(R)^{-1} d R=\infty$ then $u(x) \notin L^{2}(\Omega)$,

where

$$
\Phi^{*}(r)=\exp \left(\int_{R_{0}}^{r}(2 t)^{-1} \sup _{|x|=t}\left\{\sigma_{1}(x)+\eta_{1}(x)\right\} d t\right)
$$

Remark 8.3 (The case $A(x) \equiv E$.)

Here we consider the case $A(x) \equiv E(n \times n$ identity matrix). Then the Assumption (F.4) is satisfied automatically.

(1) If $A(x) \equiv E$, then in Theorem 8.1 and Theorem 8.2 the Assumption (E.2) can be replaced with (E.2) in $\$ 1$.

(2) If $A(x) \equiv E$ and $\sigma_{i}(x)+\eta_{i}(x)$ depends only on $r=|x|$, then in Theorem 8.1 and Theorem 8.2 we may take $b_{i}=1(i=1,2)$ so that the Assumption (F.2) reduces to (F.2) in $\S 1$. (In this case (F.3.i) is satisfied automatically and (F.3. ii) can be neglected.)

(3) If $A(x) \equiv E, B(x) \equiv O(n \times n$ zero matrix $)$ and, both $\sigma_{i}(x)$ and $\eta_{i}(x)$ depend only on $r=|x|$, then in Theorem 8.1 and Theorem 8.2 the Assumption (E.2) can be replaced (E.2)' in Remark 1.4.

\section{References}

[1] Agmon, S., Lower bounds for solutions of Schrödinger equations, J. Analyse Math., 23 (1970), 1-25.

[2] Arai, M. and Uchiyama, J., Growth order of eigenfunctions of Schrödinger oparators with potentials admitting some integral conditions II -Applications-, Publ. RIMS, Kyoto Univ., 32 (1996), 617-631.

[3] Eastham, M.S.P. and Kalf, H., Schrödinger-type operators with continuous spectra, Research Notes in Mathematics 65, Pitman, Boston, London, Melbourne, 1982.

[4] Eidus, D.M., The principle of limit amplitude, Russian Math. Surveys, 24 (1969), no. 3, 97 -167 .

[5] Kalf, H. and Kumar, V.K., On the absence of positive eigenvalues of Schrödinger operators with long range potentials, Trans. Amer. Math. Soc., 275 (1983), 215-229.

[6] Kato, T., Growth properties of solutions of the reduced wave equation with variable coefficients, Comm. Pure Appl. Math., 12 (1959), 403-425.

[7] Khosrovshahi, G.B., Levine, H.A. and Payne, L.E., On the positive spectrum of Schrödinger 
operators with long range potentials, Trans. Amer. Math. Soc., 253 (1979), 211-228.

[8] Mochizuki, K., Growth properties of solutions of second order elliptic differential equations, J. Math. Kyoto Univ., 16 (1976), 351-373.

[9] von Neumann, J. and Wigner, E.P., Über merkwürdige diskrete eigenwerte, Phys. Z., 30 (1929), 465-467.

[10] Roze, S.N., On the spectrum of an elliptic operator of second order, Math. USSR Sb., 9 (1969), 183-197.

[11] Uchiyama, J., Lower bounds of growth order of solutions of Schrödinger equations with homogeneous potentials, Publ. RIMS, Kyoto Univ., 10 (1975), 425-444.

[12] Polynomial growth or decay of eigenfunctions of second-order elliptic operators, Publ. RIMS, Kyoto Univ., 23 (1987), 975-1006.

[13] Uchiyama, J. and Yamada, O., Sharp estimates of lower bounds of polynomial decay order of eigenfunctions, Publ. RIMS, Kyoto Univ., 26 (1990), 419-449. 